\title{
The Design of the Tokamak Physics Experiment (TPX)
}

J. A. Schmidt, ${ }^{a}$ K. I. Thomassen, ${ }^{b}$ R. J. Goldston, ${ }^{a}$ G. H. Neilson, ${ }^{c}$ W. M. Nevins, ${ }^{b}$ J. C. Sinnis, ${ }^{a}$ P. Anderson, ${ }^{n}$ W. Bair,${ }^{r}$ W. L. Barr,${ }^{b}$ D. B. Batchelor,${ }^{c}$ C. Baxi, ${ }^{n}$ G. Berg, ${ }^{o}$ S. Bernabei, ${ }^{a}$ J. M. Bialek, ${ }^{a}$ P. T. Bonoli, ${ }^{h}$ A. Boozer, ${ }^{i}$ D. Bowers, ${ }^{p}$ G. Bronner, ${ }^{a}$ J. N. Brooks, ${ }^{d}$ T. G. Brown, ${ }^{q}$ R. Bulmer, ${ }^{b}$ D. Butner, ${ }^{b}$ R. Campbell, ${ }^{e}$ T. Casper, ${ }^{b}$ E. Chaniotakis, ${ }^{h}$ M. Chaplin, ${ }^{b}$ S. J. Chen, ${ }^{a}$ E. Chin, ${ }^{n}$ J. Chrzanowski, ${ }^{a}$ J. Citrolo, ${ }^{a}$ M. J. Cole, ${ }^{c}$ F. Dahlgren, ${ }^{a}$ F. C. Davis,${ }^{c}$ J. Davis, ${ }^{p}$ S. Davis, ${ }^{a}$ N. Diatchenko, ${ }^{h}$ S. Dinkevich, ${ }^{r}$ Y. Feldshteyn, ${ }^{a}$ B. Felker, ${ }^{b}$ T. Feng, ${ }^{a}$ M. E. Fenstermacher, ${ }^{b}$ R. Fleming, P. J. Fogarty, ${ }^{c}$ W. Fragetta, ${ }^{r}$ E. Fredd, ${ }^{a}$ M. Gabler, ${ }^{o}$ J. Galambos,,$c$ Y. Gohar, ${ }^{d}$ P. L. Goranson, ${ }^{c}$ N. Greenough, ${ }^{a}$ L. R. Grisham, ${ }^{a}$ J. Haines, ${ }^{p}$ S. Haney, ${ }^{b}$ W. Hassenzahl, ${ }^{b}$ J. Heim, ${ }^{b}$ P. J. Heitzenroeder, ${ }^{a}$ D. N. Hill, ${ }^{b}$ T. Hodapp, ${ }^{n}$ W. A. Houlberg, ${ }^{c}$ A Hubbard, ${ }^{h}$ A Hyatt, ${ }^{n}$ M. Jackson, ${ }^{b}$ E. F. Jaeger, ${ }^{c}$ S. C. Jardin, ${ }^{a}$ J. Johnson, ${ }^{a}$ G. H. Jones, ${ }^{c}$ D. R. Juliano, ${ }^{l}$ R. Junge, ${ }^{n}$ M. Kalish, ${ }^{a}$ C. E. Kessel, ${ }^{a}$ D. Knutson, ${ }^{a}$ R. J. LaHaye, ${ }^{n}$ D. D. Lang, ${ }^{b}$ R. A. Langley, ${ }^{c}$ S.-L. Liew, ${ }^{a}$ E. Lu, ${ }^{a}$ H. Mantz, ${ }^{p}$ J. Manickam, ${ }^{a}$ T. K. Mau, ${ }^{j}$ S. Medley, ${ }^{a}$ D. R. Mikkelsen, ${ }^{a}$ R. Miller, ${ }^{f}$ D. Monticello, ${ }^{a}$ D. Morgan, ${ }^{p}$ P. Moroz, ${ }^{k}$ C. Motloch,,${ }^{g}$ J. Mueller, ${ }^{q}$ L. Myatt, ${ }^{h}$ B. E. Nelson, ${ }^{c}$ C. L. Neumeyer, ${ }^{r}$ D. Nilson, ${ }^{b}$ T. O'Conner, ${ }^{b}$ L. D. Pearlstein, ${ }^{b}$ W. A. Peebles, ${ }^{j}$ M. Pelovitz, ${ }^{a}$ F. W. Perkins, ${ }^{a}$ L. J. Perkins, ${ }^{b}$ D. Petersen,,${ }^{b}$ R. Pillsbury, ${ }^{h}$ P. A. Politzer, ${ }^{n}$ N. Pomphrey, ${ }^{a}$ M. Porkolab, ${ }^{h}$ A. Posey, ${ }^{b}$ A. Radovinsky, ${ }^{h}$ S. Raftopoulis, ${ }^{a}$ S. Ramakrishnan, , J. Ramos, ${ }^{h}$ W. Rauch, ${ }^{a}$ D. Ravenscroft, ${ }^{b}$ K. Redler, ${ }^{n}$ W. T. Reiersen, ${ }^{a}$ A. Reiman, ${ }^{a}$ E. Reis, ${ }^{n}$ G. Rewoldt, ${ }^{a}$ D. J. Richards, ${ }^{c}$ R. Rocco, ${ }^{r}$ T. D. Rognlien, ${ }^{b}$ D. Ruzic, ${ }^{l}$ S. Sabbagh, ${ }^{m}$ J. Sapp,${ }^{p}$ R. O. Sayer, ${ }^{c}$ J. E. Scharer, ${ }^{k}$ L. Schmitz, ${ }^{j}$ J. Schultz, ${ }^{h}$ L. Sevier, ${ }^{n}$ S. E. Shipley, ${ }^{c}$ R. T Simmons, ${ }^{a}$ D. Slack, ${ }^{b}$ G. R. Smith, ${ }^{b}$ R. Stambaugh, ${ }^{n}$ G. Steill, ${ }^{a}$ T. Stevenson, ${ }^{a}$ S. Stoenescu, ${ }^{r}$ K. T. St. Onge, ${ }^{c}$ D. P. Stotler, ${ }^{a}$ T. Strait, ${ }^{n}$ D. J. Strickler, ${ }^{c}$ D. W. Swain, ${ }^{c}$ W. Tang, ${ }^{a}$ M. Tuszewski, ${ }^{f}$ M. A. Ulrickson, ${ }^{a}$ A. VonHalle, ${ }^{a}$ M. S. Walker, ${ }^{c}$ C. Wang, ${ }^{m}$ P. Wang, ${ }^{h}$ J. Warren, ${ }^{r}$ K. A. Werley,${ }^{f}$ W. P. West, ${ }^{n}$ F. Williams, ${ }^{p}$ R. Wong, ${ }^{b}$ K. Wright, ${ }^{a}$ G. A. Wurden, ${ }^{f}$ J. J. Yugo, ${ }^{c}$ L. Zakharov, ${ }^{a}$ and J. Zbasnik ${ }^{b}$

\footnotetext{
The Tokamak Physics Experiment is designed to develop the scientific basis for a compact and continuously operating tokamak fusion reactor. It is based on an emerging class of tokamak operating modes, characterized by beta limits well in excess of the Troyon limit, confinement scaling well in excess of $\mathrm{H}$-mode, and bootstrap current fractions approaching unity. Such modes are attainable through the use of advanced, steady state plasma controls including strong shaping, current profile control, and active particle recycling control. Key design features of the TPX are superconducting toroidal and poloidal field coils; actively-cooled plasma-facing components; a flexible heating and current drive system; and a spacious divertor for flexibility. Substantial deuterium plasma operation is made possible with an in-vessel remote maintenance system, a lowactivation titanium vacuum vessel, and shielding of ex-vessel components. The facility will be constructed as a national project with substantial participation by U.S. industry. Operation will begin with first plasma in the year 2000 .
} 


\section{INTRODUCTION}

The Tokamak Physics Experiment (TPX) will play the important program role of determining whether the tokamak approach can evolve smaller, less expensive, and more attractive fusion reactors than are forecast using conventional physics rules. TPX is being designed to extend advanced tokamak operating modes with high beta, confinement, and bootstrap current fraction to the steady-state regime. While the mission of TPX is primarily to explore advanced physics regimes in the steady state, doing so will have the added benefit of expanding our tokamak technology base. Key technology developments will be in the use of superconducting magnets; stcady state power handling in the divertors, low activation material usage to allow access to in-vessel components during early phases of operation, and in remote maintenance techniques.

In September of 1991, a Task Force on Energy Priorities of the Secretary of Energy Advisory Board (SEAB) challenged the fusion program to chart a course toward improving the tokamak, while endorsing ITER as the vehicle for carrying out the burning plasma mission. A National Task Force (NTF) was established by the fusion community in October 1991 to provide guidance to the new initiative on issues of organization, mission, and design. In addition, a subpanel of the Fusion Energy Advisory Committee (FEAC Panel 2) was chartered in November of that year to recommend to FEAC the proper course for the new initiative. In March and April 1992 the NTF and FEAC 2 endorsed the steady state/advanced tokamak mission for the Tokamak Physics Experiment. In May of that year the NTF endorsed the project's choice of superconducting technology for the TPX magnets. From May through September, key decisions on the major tokamak parameters, configura-

\footnotetext{
aPrinceton Plasma Physics Lab, Princeton, New Jersey.

${ }^{b}$ Lawrence Livermore National Lab, Livermore, California.

cOak Ridge National Lab, Oak Ridge, Tennessee

${ }^{\circledR}$ Argonne National Lab, Argonne, Illinois.

'Sandia National Lab, Albuquerque, New Mexico.

Los AJamos National Lab, Los Alamos, New Mexico.

sIdaho National Engineering Lab, Idaho Falls, Idaho.

"Massachusetts Institute of Technology, Cambridge, Massachusetts.

${ }^{i}$ College of William and Mary, Williamsburg, Virginia.

University of California at Los Angeles, Los Angeles, California.

"University of Wisconsin, Madison, Wisconsin.

University of Illinois at Urbana-Champaign, Urbana, Illinois.

mColumbia University, New York, New York.

"General Atomics, San Diego, California.

${ }^{\circ}$ Rockwell International, Canoga Park, California.

${ }^{p}$ McDonnell Douglas Missile Systems, St. Louis, Missouri.

${ }^{\circ}$ Grumman Space \& Electronic Div., Princeton, New Jersey.

rEbasco Services, New York, New York.
}

tion, heating systems, and cost objectives were made. These decisions were reviewed and endorsed by the $\mathrm{Na}$ tional TPX Council, an oversight committee chartered to participate in decisions relating to programmatic and managerial aspects of the Project. In September 1992, the SEAB Task Force concluded that the proposed TPX facility was responsive to their challenge of the previous year and reiterated an earlier recommendation for 5\% real annual growth in the fusion budget over several years. Upon their recommendation, the Department of Energy approved the proposed mission for TPX and authorized the conceptual design to proceed.

A Conceptual Design Review (CDR), coupled with a Department of Energy cost review, are critical steps in the proccss leading to full project approval. The CDR was conducted in March 1993, by an international team of fusion experts. The purpose of the CDR, as expressed in DOE's charge, was "to obtain expert advice on the physics and engineering design of TPX, and on the project's cost estimates, schedules, and management arrangements." Because the review encompassed more areas (most notably environment, safety, health, and cost, schedule management) than typical fusion program reviews, the panel was large ( 32 members) and included both field (five universities, seven U.S. national laboratories, and eight foreign national laboratories) and DOE (four Office of Fusion Energy and eight from other offices) personnel. The significant percentage of foreign CDR Committee members $(25 \%)$ reflected the international character of the magnetic fusion program. The general findings of the CDR Committee were as follows:

- The conceptual design work has been of very high quality.

- The TPX conceptual design project has been laudably constituted and run as a national magnetic fusion project with significant involvement of several DOE laboratories (primarily Lawrence Livermore National Laboratory, Oak Ridge National Laboratory, Plasma Fusion Center of the Massachusetts Institute of Technology, and Princeton Plasma Physics Laboratory) and universities.

- The TPX is an excellent vehicle for developing advanced-tokamak steady-state concepts which, together with burning plasma and nuclear testing results from ITER, should lead to a compact, efficient demonstration reactor.

- The project scope and tools provided (profile control, shaping capability, divertor) are sufficient for the TPX mission and the machine size is an [appropriate] trade-off between the desire 
for higher performance and the greater cost involved.

- The TPX conceptual design is technically sound and supports the project's mission.

\subsection{Design Approach}

The physics design of TPX makes extensive use of the criteria and analytical tools used in the BPX, ITER (CDA), and earlier mainline tokamak design studies. However, consistent with its role to improve the tokamak, it also incorporates more innovative features, borrowed from recent "advanced" reactor studies (ARIES, SSTR), and it capitalizes on recent promising developments in tokamak experiments and theory.

The engineering design strategy is to limit the required technology development and to employ conservative engineering design criteria. This design basis is consistent with the central role of this project in the U.S. fusion program and the associated need for an expeditious design and construction schedule.

The configuration and technology choices related to the TPX design are driven by its mission. The choice of superconducting technology for the magnets clearly derives from the requirement for long pulse, high-duty factor operation. The range of plasma configurations that can be accommodated and the array of heating systems selected, derive from the advanced tokamak mission element. The requirement for TPX to operate with deuterium as a fuel results in a neutron fluence that has a strong impact on the configuration and maintenance. The requirement to operate with deuterium derives in part from the significant increase in plasma and neutral beam performance associated with this fuel. In addition, experiments have shown that deuterium offers more favorable access to advanced regimes, providing a crucial element of physics flexibility that would be lacking in a hydrogen-only machine. Short-pulse DT operation will also be available ncar the end of the operating life to study alpha particle physics in advanced regimes. Shielding is required between the plasma and the TF coils to reduce the nuclear heating in the magnets and the associated refrigeration requirements. This shielding facilitates the use of hands-on maintenance outside the shield although remote maintenance of hardware inside the vacuum vessel will be required after the first few years of operation.

The plan is to site the TPX facility (Fig. 1) at Princeton Plasma Physics Laboratory, in the TFTR test cell. TPX will make use of existing TFTR hardware such as power supplies, neutral beams, If heating systems, diagnostics, and water cooling. An Environmental As-

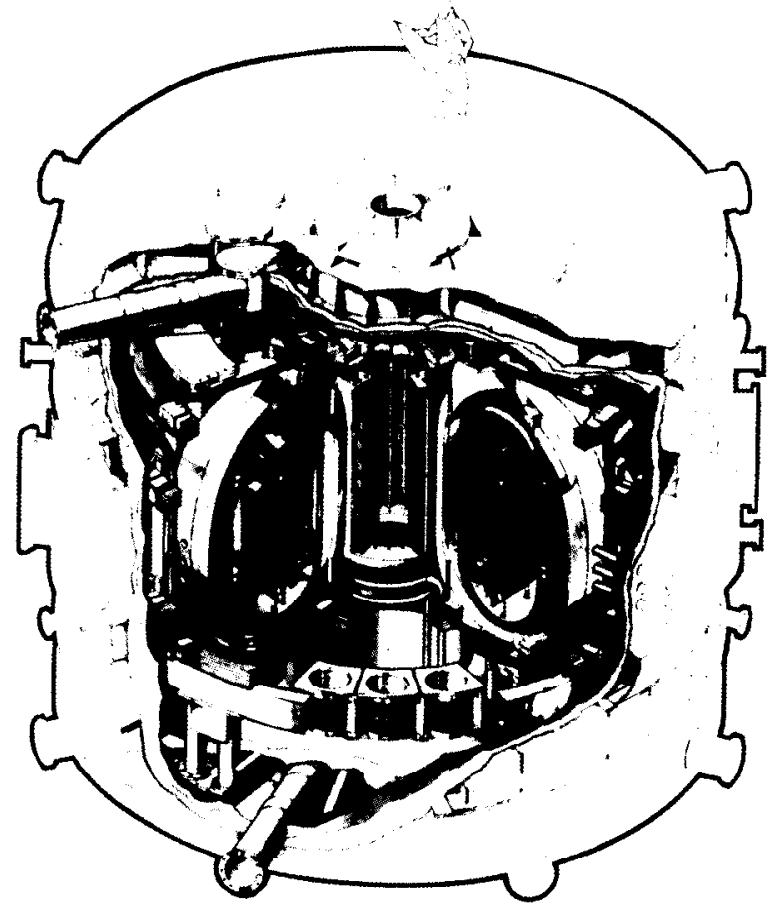

Fig. 1. The high aspect ratio, superconducting TPX machine.

sessment (EA) has been prepared that covers both the decontamination and decommissioning of TFTR and the construction and operation of TPX. The environmental impact of the construction and operation of TPX bears many similarities to these phases of the TFTR project. This is due primarily to the fact that TPX will be constructed in the TFTR test cell, use many of the TFTR support systems and will be constrained to operate within TFTR site limits. The primary conclusion documented in the EA is that "no adverse long-term impacts would result from either project, either separately or combined." If the review process leads to the concurrence of DOE with the EA conclusions, then a Finding of No Significant Impact (FONSI) could be expected early in 1994.

The TPX Project is a focused, national effort involving the coordinated resource of a large part of the DOE's fusion program. Although PPPL is responsible for the project, the Project includes, as participants, many of the U.S. plasma physics research laboratories, universities, and industrial firms. In addition to the subsystems that will be assigned to industry for design and the industrial systems integration and construction management, essentially all of the fabrication will be carried out by industry. The industrial involvement will be through 
free and open competitive procurements. A Program Advisory Committee (PAC) was formed in December 1992, to review proposed plans and schedules for experimental research on the TPX facility. During the design and construction phase of the project, the PAC will report to the Program Director and will work with the TPX physics team in setting details of the project physics requirements.

An extensive set of design documents describes the design requirements, physics basis, subsystem design, and $R \& D$ needs. In this paper we provide an overview of the conceptual design of TPX, as detailed in these documents.

\section{MISSION}

In preparation for building an attractive fusion Demonstration (DEMO) power plant in the twenty-first century several parallel and essential paths of research and development are being pursued in the world fusion programs. Depicted in Fig. 2, these can be grouped into devices and programs which push the frontiers of high performance and explore DT operation in tokamaks, programs and facilities for carrying out the vital nuclear engineering and materials aspects of the program, and those programs and experiments devoted to fusion reactor "concept improvement." The last of these include activities in the "core" program that seek fundamental knowledge of plasmas and that explore limits in our push towards physics regimes leading to more attractive reactors.

Attractive features based on physics performance include higher power density, steady state operation, and

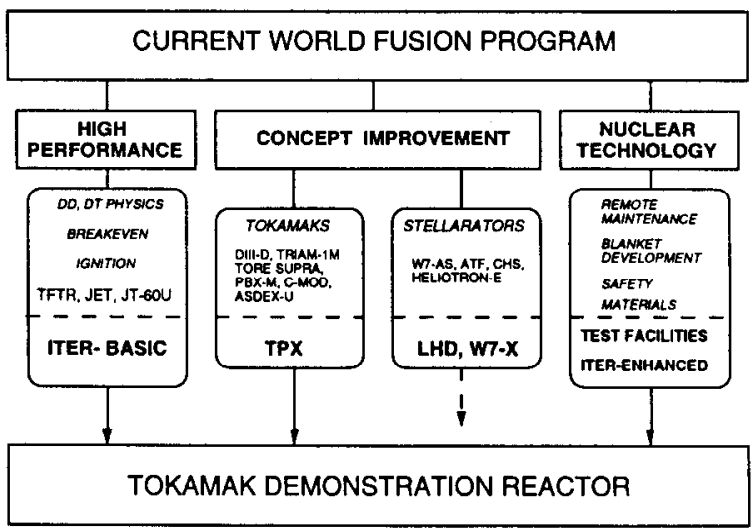

Fig. 2. Major parallel tasks for fusion power development leading to a demo. smaller unit size (and therefore capital cost). TPX is the centerpiece of a program to provide the tokamak physics data base for these attractive features through exploration of advanced modes of operation in the steady state. The results should complement those from the basic phase of International Thermonuclear Experimental Reactor (ITER), on the performance of ignited plasmas, and on controlled burning. Moreover, TPX will make substantial contributions to the ultimate success of ITER. TPX is the only steady-state tokamak planned in the world program that can develop and test operating regimes that address the issues of fully non-inductive, high-bootstrap current drive, combined with reactor-relevant divertor conditions. The ability to accommodate current-drive upgrades for steady-state operation and major modifications to the divertor system are being incorporated into the ITER design. The capability of TPX for flexible experimentation will provide very valuable guidance in optimizing the operations of ITER, and in the choice of future upgrades. By the same token, studies performed in the later phases of ITER will provide stepping-stones for advanced regimes developed in TPX to be tested, at least in part, for application in an economic, compact, steady-state demonstration power reactor.

These two complementary machines should provide the experimental basis for choosing an optimum reactor direction. A DEMO based on the present ITER direction would have inductively driven current and ignited plasmas, in a low aspect ratio tokamak, with conservative performance assumptions (hence larger unit size). The TPX-based approach, using a current-driven high aspect ratio tokamak, would control current profiles to achieve superior performance (hence smaller unit size) in steady state. Data from both TPX and ITER are necessary to make an optimum reactor choice.

\subsection{TPX Mission Statement}

The mission of the Tokamak Physics Experiment (TPX) is to develop the scientific basis for an economic, compact, and continuously operating tokamak fusion reactor.

Supporting objectives are to:

- Optimize plasma performance through active control of the current profile and of plasma-wall interactions, and by advanced plasma shapingleading to a compact tokamak fusion reactor.

- Achieve this optimization using techniques for non-inductive current drive and profile control that are consistent with efficient continuous operation of a tokamak fusion reactor. 
- Demonstrate the integration of optimized plasma performance and efficient continuous operation in fully steady state tokamak plasmas.

Recent experimental and theoretical results indicate that the key TPX mission elements of advanced tokamak and steady-state operation are highly complementary. The current-profile control that can be made available in a fully current-driven tokamak is a key control element for improving both plasma pressure limits and energy confinement. The recycling control that will be available with a pumped divertor operating in the presence of a fully equilibrated vacuum vessel wall should allow direct influence over the plasma edge conditions that have proven to be so crucial to enhanced confinement regimes. Thus the combination of the steady-state and advanced tokamak themes in a single experimental device is natural. At the same time it should be recognized that while success in both of these areas will provide the greatest benefits from TPX, advances in either alone will still be very beneficial to the development of economic fusion power.

\subsection{Physics Objective of TPX}

In the context of the mission of TPX, a set of ambitious experimental objectives has been established for the TPX program. These are:

- Achievement of $\beta_{N}{ }^{*}=5-6$ and $H=3-4$, at $q_{95}$ $\sim 4\left[\right.$ where $\beta_{N}{ }^{*} \equiv \beta^{*} /(I / a B)$ with the asterisk indicating the use of RMS pressure; and $H \equiv \tau_{\mathrm{E}} / \tau_{\mathrm{L}}$. mode, the confinement enhancement factor over L-mode].

- Demonstration of efficient current drive, extrapolating to $Q_{\mathrm{cd}}=15-30$ in a steady-state reactor, via high bootstrap fraction [where $Q_{\mathrm{cd}} \equiv P_{\text {fus }} P_{\mathrm{cd}}$ ].

- Demonstration of advanced power and particle exhaust, extrapolating to $\sim 3 \mathrm{MW} / \mathrm{m}^{2}$ neutron power flux and $\sim 10 \% \mathrm{He}$ ash in a steady-state tokamak reactor.

- Attainment of high plasma and component reliability: $\sim 80 \%$ availability of all components, and $\sim 1$ disruption/10 hours.

These experimental objectives, which are to be met simultaneously in steady state, flow from the TPX mission and from an understanding of the impact of advances in tokamak physics on projected reactor performance.

Simultaneous goals of high confinement and high normalized plasma pressure have been set because these are both required in order to reduce the unit size of a tokamak reactor. The fusion gain of reactor, proportional to $\left\langle n^{2} T^{2}\right\rangle \tau /\langle n T\rangle$, scales approximately as $\left(\beta^{*} H I_{\mathrm{p}} R / a \beta\right)^{2}$. Peaking of the profiles enhances fusion power output at fixed $\beta$, so $\beta^{*} / \beta$ ( 1.25 for typical profiles) is a good overall measure for this enhancement. If we now require a specified gain in order to obtain very high $Q$, and fix $q^{*}$ (the cylindrical safety factor at the plasma edge), we have:

$$
\frac{a\left(1+\kappa^{2}\right) B}{q^{*}} \infty \frac{\beta}{\beta^{*} H}
$$

Thus, improvements in the parameter $H^{*} \equiv H \beta^{*} / \beta$ can lead to reduction in reactor size and/or field. However, neutron wall loading must be specified at a value that is both economically attractive and technically achievable (e.g., $-3 \mathrm{MW} / \mathrm{m}^{2}$ ). At fixed wall loading, then, the machine size and field are constrained by:

$$
\frac{\left(1+\kappa^{2}\right) \kappa^{1 / 4} a^{1 / 2} B^{2}}{q^{*}(R / a)} \propto \frac{1}{\beta_{N}^{*}}
$$

Thus, size and/or field can only be decreased at fixed wall loading if $\beta_{N}{ }^{*}$ is increased.

The requirement for current-drive power in steady state, at fixed temperature, scalcs as:

$$
\frac{P_{\mathrm{cd}}}{P_{\text {fus }}} \propto \frac{\left(1-f_{\mathrm{bs}}^{*}\right) n I_{\mathrm{p}} R / \eta_{\mathrm{cd}}}{\left(\beta_{N}^{*} l_{p} / a B\right)^{2} B^{4} R a^{2} \kappa} \propto \frac{\left(1-f_{\mathrm{bs}}^{*}\right)}{\eta_{\mathrm{cd}}} \frac{\beta_{N}}{\left(\beta_{N}^{*}\right)^{2} a \kappa B}
$$

where $f_{\mathrm{bs}}{ }^{*} \propto q^{*}(R / a)^{1 / 2} \beta_{N}$ corrcsponds to the effective useful bootstrap current fraction (taking into account the fact that bootstrap currents that flow in the wrong region of the plasma are undesirable), and $\eta_{\mathrm{CD}}$ denotes the current drive efficiency, $n I_{\mathrm{P}} R / P$, with external heating systems. Thus high values of $H^{*}$ and $\beta_{N}{ }^{*}$ can allow a steadystate fusion reactor with reduced recirculating power, smaller unit size, and lower field, current, stored energy, and heat flux, implying lower cost and higher reliability. For these reasons, the simultaneous achievement of high $\beta_{N}{ }^{*}$, high $H^{*}$, and high bootstrap fraction must be key experimental goals for TPX.

Our systems-code studies indicate that if $\beta_{N}$ can be increased from 2.5 to 6 and $\mathrm{H}$ from 2 to 3 (for a particular set of density and temperature profile assumptions), then the Cost of Electricity (COE) from a demonstration tokamak reactor, at fixed unit size, drops by about a factor of two. The minimum unit size of a reactor at a given COE drops by about a factor of four (e.g., from $2000 \mathrm{MWe}$ to $500 \mathrm{MWe}$ ). The combination of reduced $\mathrm{COE}$, reduced unit size, and fully continuous operation would make the tokamak much more attractive in the world energy market. As the size of a reactor is reduced at fixed neutron wall-loading, the parallel heat flux to the divertor, as measured by $P / R$, decreases. Further- 
more, if the field or size is reduced, the impact of disruptions is also strongly ameliorated. Thus, achievement of the first two of the TPX experimental objectives constitutes a key step in the development of an attractive tokamak.

In order to achieve a level of neutron wall loading that is optimum in terms of first-wall life and blanket economics, tokamak divertors will need to operate in advanced gaseous/radiative modes that disperse the associated plasma heat flux over a wide area. At the same time the divertor must maintain good plasma purity and helium exhaust while controlling the density for efficient current drive. These requirements set the TPX experimental objectives on power and particle exhaust.

Finally, the achievement of true steady state clearly requires the minimization of plasma disruptions. The TPX objective of 1 disruption per 10 hours of plasma operation has been set in order to establish the level of plasma reliability needed for steady-state tokamak operation. Even though the destructive impact of disruptions will be considerably reduced in a compact reactor, techniques to minimize damage to divertors and first-wall components need to be developed as well.

\subsection{Physics Criteria for TPX}

The physics design criteria for TPX flow from its mission and experimental objectives. The design features and performance parameters required to meet the TPX goals are described below.

Long pulse lengths are required to assure that processes such as current profile evolution and plasmawall interaction are studied fully in equilibrium. Since TPX will be used to develop and demonstrate advanced plasma control techniques, the pulse lengths must be long enough to eliminate the dependence on initial conditions. One key time constant is the global currentrelaxation time, which can be in the range of $\sim 100$ seconds. A much longer pulse than this is required to demonstrate full control for many characteristic times. Consensus estimates of the time required for the plasma and wall to come into equilibrium are in the few $100 \mathrm{~s}$ of seconds range. High duty factor is required in TPX in order to test erosion and redeposition rates in divertors and first-wall components, and to demonstrate high plasma reliability, requiring $\geq 50$ hours of disruption-free operation late in the life of the machine. Thus the baseline TPX facility is designed for 1000 -s pulses, while the tokamak itself is designed for full steady-state operation so that the pulse length can be extended beyond $1000 \mathrm{~s}$ solely by upgrading auxiliary systems.
An advanced, flexible divertor configuration is required to meet the power and particle exhaust objectives of TPX. Since enhanced confinement modes are sensitive to edge conditions, the TPX divertor must be designed to encourage strong localized recycling in the divertor channel, while severely limiting recycling back to the main plasma. In combination with active, variable pumping, the divertor system must provide control over the plasma density and boundary conditions, and must support the divertor plasma cooling required for effective heat dispersal. A large volume in the vacuum vessel and the flexibility to modify the configuration are essential to permit tests of alternate configurations as well. Heating system upgrade capability to $P_{\text {aux }} / A_{\text {sep }}=0.75 \mathrm{MW} /$ $\mathrm{m}^{2}$, corresponding to the heat flux across the separatrix of a tokamak reactor with $3 \mathrm{MW} / \mathrm{m}^{2}$ neutron flux, is provided to permit divertor tests at DEMO-relevant heat fluxes.

The poloidal field coils and divertor configuration must allow advanced tokamak modes covering a large operating window in beta and internal inductance, $l_{\mathrm{i}}$, to be explored. Experiments on DIIl- $D^{1}$ suggest that high triangularity, $\delta_{x}$, and a double-null poloidal divertor configuration are required for the best plasma performance. Theoretically, access to second-stability in the outer region of the plasma is made possible by high triangularity. Thus a high value of $\delta_{x}$ is a natural requirement for an advanced tokamak experiment. The poloidal field system must strike the balance between the requirement for shape flexibility and the need for a constrained slot divertor by leaving the inner strike point, which sees little heat and particle flux, free to move while rigorously maintaining the slot configuration for the outer strike point. A double-null design achieves this balance because of the substantial reduction in the inner divertor heat loads.

The TPX is designed as a high-aspect-ratio tokamak. A number of studies ${ }^{2}$ have pointed to the advantages of higher aspect ratios $\sim 4-5$ for steady-state tokamak reactors that make use of the bootstrap current to reduce the recirculating power. Moreover, high-aspect-ratio data from TPX are needed to supplement the data from present and planned lowcr-aspect-ratio devices in order to provide the information needed to make an informed

\footnotetext{
${ }^{1}$ E. A. Lazarus and the DIII-D Team (1993). Dependence of $\beta-\tau$ on plasma shape in DIII-D. Presented at 20th EPS Conference on Controlled Fusion and Plasma Physics, July 26-30, Lisboa, Portugal. To be published in Proceedings.

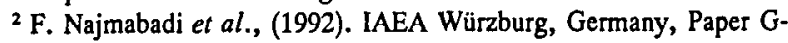
1-1-1(R); J. Wesley et al., (1992). IAEA Würzburg, Germany, Paper F-1-2; Y. Seki et al., (1991). Rep. JAERI-M 91-081, JAERI, Naka.
} 
choice on the optimal aspect ratio for DEMO. It is worth noting in this context that high-aspect-ratio data will also be required for very-long-pulse inductive devices. ${ }^{3}$

The heating and current drive system must provide the detailed current-profile control required for studies of advanced tokamak configurations in steady state. It is also important to heat both ions and electrons in order to simulate a reactor where $T_{i} \approx T_{e}$. Neutral beam injection provides ion heating and bulk current drive. Fast waves provide electron heating and centrally-peaked current drive. Lower hybrid waves heat electrons, and through active launch-spectrum control, permit control over the driven current profile. The TPX facility provides enough power in each of these systems to allow the necessary studies of steady-state and advanced-tokamak issues. Electron cyclotron heating and current drive can be added for detailed current profile control and electron heating.

The performance paramcters for TPX, i.e., the size, field strength, and heating power, are set by the requirements for advanced physics studics. Since a key mission of TPX is to learn to take advantage of a high bootstrap current fraction as a means of reducing the recirculating power in a steady-state reactor, it is very important that the bootstrap current profile in TPX be representative of what is anticipated in a tokamak reactor. Quantitatively we consider it acceptable if the collisionality is such that the total bootstrap current is reduced by $\leq 15 \%$ from its fully collisionless value. For a given set of tokamak parameters $(I p, B, R, q, a)$ and a given $\beta_{N}$, the requirement for a reactor-prototypical bootstrap current profile sets an upper limit on the density operating point, which we denote $n_{\text {bs }}$.

$$
n_{e}<n_{b s} \equiv C_{1} \beta_{N}\left(\nu^{*}{ }_{\text {req }}\right)^{1 / 3} I_{p} 2 / 3 B^{2 / 3} /\left(R^{5 / 6} q^{1 / 3} a^{1 / 6}\right)
$$

where we have used only the definitions of $\beta_{N}$ and $\nu^{*}$ to define the scaling of $n_{\mathrm{bs}}$.

The second performance-related requirement is that the fast electrons used for lower-hybrid current drive be well-localized in order to ensure effective current profile control. Assuming that the diffusivity of fast electrons is comparable to the bulk electron thermal diffusivity (an assumption confirmed by experimental results $)^{4}$, the requirement that the fast electrons diffuse minimally before they slow down sets a limit on $\tau_{s e} / \tau_{E}$, or equivalently on $n \tau_{\mathrm{E}}$. Quantitatively, to obtain reasonably well localized current-drive we require $\tau_{\mathrm{se}} / \tau_{\mathrm{E}} \leq 0.13$. If we take $n \tau_{\mathrm{E}} T \propto\left(H I_{\mathrm{P}} R / a\right)^{2} A_{i}$ and substitute for $T$ in terms of $\beta_{N}$,

${ }^{3} \mathrm{~N}$. Inoue et al., (1992). IAEA Würzburg, Germany, Paper G-1-4. 4S. Texter et al., (1993). Phys. Lett A 175, 428; R. Kaita et al., (1992). IAEA Würzburg, Germany, PBX-M post-deadline paper. we arrive at a requirement that $n_{\mathrm{c}}$ must be above some critical density, $n_{\mathrm{LH}}$.

$$
n_{e}>n_{\mathrm{LH}} \equiv C_{2} \beta_{\mathrm{N}}\left(n \tau_{\mathrm{E}}\right)_{\mathrm{req}} a B /\left(H^{2} A_{i} I_{p} R^{2}\right)
$$

The requirement $n_{\mathrm{LH}}<n_{\mathrm{c}}<n_{\mathrm{bs}}$ constitutes a density operating window for advanced tokamak studies. Figure 3 shows this operating window for TPX at $I_{P}=1.5$ MA, $\beta_{N}=3.3, H=3$. The ratio $n_{\mathrm{bs}} / n_{\mathrm{LH}}$, the width of this window, is a key "figure of merit" for an advanced tokamak experiment. If we scale $q \propto(a / R)^{1 / 2}$ for fixed bootstrap fraction at a given $\beta_{N}$, and we use $I_{3} \equiv I_{p}$ when $q_{95}=3$ to gather terms, we obtain:

$$
n_{\mathrm{bs}} n_{\mathrm{LH}} \propto H^{2} A_{i} I_{3}^{5 / 3}(R / a)^{13 / 6} / B^{1 / 3}
$$

The major parameters of TPX have been chosen such that the requirements on the bootstrap collisionality correction and $\tau_{\mathrm{sc}} / \tau_{\mathrm{E}}$ are met over a wide range of conditions. In deriving this scaling, we have assumed that the confinement time scales as the square root of the atomic mass $A_{i}$. On this assumption the size and/or magnetic field, as well as the heating power, of a hydrogenonly device would have to be substantially increased relative to TPX to have the same advanced tokamak performance. More fundamentally, however, enhanced confinement modes are much more reliably obtained in deuterium than hydrogen. Thus the ability to operate substantially in deuterium is a key requirement.

\subsection{Technology in TPX}

While the program role and emphasis is on the physics objectives of TPX, many of its supporting technologies are in the mainstream of fusion reactor development. These include superconducting toroidal and poloidal magnets using $\mathrm{Nb}_{3} \mathrm{Sn}$ cable-in-conduit conductor, actively cooled divertors, steady state auxiliary heat-

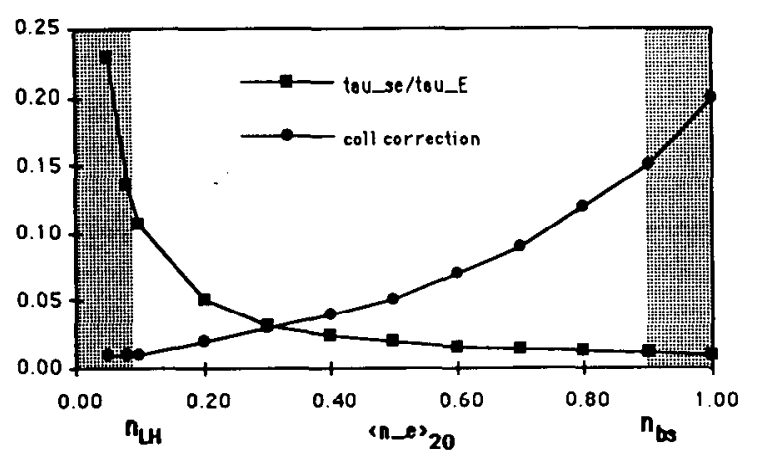

Fig. 3. TPX density operating window at $I_{\mathrm{p}}=1.5 \mathrm{MA}, \beta_{N}=3.3$, $H=3$. 
ing and current drive systems, steady fueling and particle removal systems, and a system of active and passive plasma control devices.

Heat loads to the divertor surfaces are to be reduced, by a large factor, below their unattenuated values through the use of radiative or gas target schemes. Nonetheless actively cooled surfaces are employed, manufactured to high quality control standards and aligned to high precision. They will withstand heat loads of up to $15 \mathrm{MW} / \mathrm{m}^{2}$.

Deuterium operation at high performance for many long pulses (approximately $2 \times 10^{5}$ seconds per ycar, or $6 \times 10^{21} \mathrm{DD}$ neutrons per year) requires full remote maintenance in the interior of the vessel. A low activation (titanium) vacuum vessel delays, for $\sim 2$ years, the time when full internal maintenance must be initiated. Furthermore it allows a strategy where major hands-on reconfigurations or repairs are possible without a multiyear wait for activation levels to fall.

External hands on maintenance will be allowed, owing to the extensive shielding between the vessel and the toroidal magnets and around all ducts and ports. Such a level of shielding is also sufficient for full performance DT shots lasting several seconds, limited by nuclear heating in the magnets.

\section{PHYSICS DESIGN}

The design of the TPX device and facility is based on requirements developed from both physics and engineering considerations. These follow from the mission and supporting objectives for the TPX program. The steady state aspect of the mission is clearly reflected in the choice of superconducting magnets and actively-cooled in-vessel components. The "advanced tokamak" aspect of the mission is reflected in the incorporation of features associated with optimum plasma operation, such as a double-null plasma configuration with strong shaping and flexibility, a flexible heating and current drive system, active particle control, and deuterium plasma capability.

The TPX has been designed to be a step toward an attractive steady-state fusion reactor. The configuration and performance parameters of TPX were chosen so as to take advantage of the bootstrap effect in supplying a large fraction of the toroidal plasma current. This is necessary for a steady state tokamak reactor, where the recirculating power to support current drive must be minimized. The TPX divertor design accommodates advanced operating techniques for handling heat and particle loads that will be applicable to reactors. The use of a fully superconducting magnet system in TPX will provide important reactor-relevant design and operating experience. Long-pulse deuterium discharges introduce requirements for shielding, remote maintenance, and reduced-activation materials that constitute an important technological step toward a reactor. The capability to upgrade for short-pulse DT physics studies is retained.

\subsection{Major Parameters and Operating Modes}

\subsubsection{Tokamak}

The major parameters of the baseline TPX facility are summarized in the "Baseline" column of Table I. The toroidal field, plasma current, and size provide sufficient performance, in deuterium plasmas, for advanced tokamak physics studies. Reference operating scenarios have been developed based on these parameters and the planned complement of heating and current drive systems. They demonstratc the various attributes required for advanced tokamak operation: high beta, high bootstrap fraction, good fast electron confinement, and low collisionality.

To support the steady-state mission, the TPX tokamak device is designed with no inherent limitations on pulse length. This philosophy is reflected in the use of all superconducting coils, actively-cooled in-vessel components, and particle exhaust with external pumps. The baseline pulse length requirement of $1000 \mathrm{~s}$ for the total

Table I. Major Parameters of TPX

\begin{tabular}{|c|c|c|c|}
\hline & & Baseline & Maximum $^{a}$ \\
\hline Toroidal field, $B_{\mathrm{T}}$ & $\mathrm{T}$ & 4.0 & \\
\hline Plasma current, $I_{\mathrm{P}}$ & MA & 2.0 & \\
\hline Major radius, $R_{0}$ & $\mathrm{~m}$ & 2.25 & \\
\hline Aspect ratio, $R / a$ & & 4.5 & \\
\hline Elongation, $\boldsymbol{k}_{\boldsymbol{x}}$ & & 2.0 & \\
\hline Triangularity, $\delta_{x}$ & & 0.8 & \\
\hline Configuration & & $\begin{array}{l}\text { Double-null } \\
\text { poloidal divertor }\end{array}$ & $\begin{array}{l}\text { Double or single-null } \\
\text { poloidal divertor }\end{array}$ \\
\hline \multicolumn{4}{|c|}{ Heating and current drive } \\
\hline Neutral beam & MW & 8 & 24 \\
\hline Ion cyclotron & MW & 8 & 18 \\
\hline Lower hybrid & MW & 1.5 & 3.0 \\
\hline Electron cyclotron & MW & -- & 10 \\
\hline Plasma species & & $\begin{array}{l}\text { Hydrogen } \\
\text { or deuterium }\end{array}$ & Tritium \\
\hline Pulse length & s & 1000 & $>>1000$ \\
\hline
\end{tabular}

- Upgrade capabilities accommodated by the baseline design. 
facility is ample for current-profile equilibration (about 50 skin times) and significantly longer than the pulse lengths that existing high-power divertor tokamaks (e.g., DIII-D, JET, JT-60U, ASDEX-U) will achieve. Plasmawall equilibration times are more difficult to predict; it is not even clear whether the wall will saturate in longpulse operation with active pumping. In the future, the pulse length can be extended as needed beyond $1000 \mathrm{~s}$ through facility improvements that remove the limits imposed by external systems, such as cryopump and steadystate cooling capacities.

The advanced tokamak mission places special requirements on the plasma geometry. While some advanced-regime experiments have been conducted in circular plasmas, high values of elongation and triangularity were chosen for TPX because MHD stability theory and most experiments indicate that a strongly shaped cross section with a double-null (DN) poloidal divertor is optimum for such studies. The DN configuration facilitates the formation of a high-triangularity plasma when using an external poloidal field (PF) coil set and minimizes the heat loads to the inboard divertor targets. With additional power supplies, the TPX can also produce single-null (SN) plasmas to allow DN-SN comparisons under advanced plasma and divertor operating conditions. The choice of the aspect ratio $R_{0} /$ a of 4.5 was motivated by reactor studies ${ }^{5}$ that have found potentially attractive design points at aspect ratios in this range, where the bootstrap current substantially reduces auxiliary current drive requirements. The TPX will greatly expand the tokamak physics database in the high aspect ratio regime.

\subsubsection{Heating and Current Drive}

The TPX heating and current drive system serves several purposes: to heat the plasma to high temperature and normalized beta, to supplement the bootstrap contributions to the steady-state toroidal plasma current, and to control the plasma current profile. The TPX system is based on multiple technologies (neutral beams, ICRF, and lower hybrid) to best serve these diverse purposes and to provide an important element of plasma control flexibility. The combined baseline system will enable TPX to reach the beta limit at full field and plasma

\footnotetext{
${ }^{5}$ M. Kikuchi, (1990). Nucl. Fusion 30, 265; and R. W. Conn, F. Najmabadi et al., (1990). ARIES-I, A Steady-State First-Stability Tokamak Reactor with Enhanced Safety and Environmental Features, 13th Int. Conference on Plasma Physics and Controlled Nuclear Fusion Research, Washington, D.C., October 1-6, paper IAEA$\mathrm{CN}-53 / \mathrm{H} \cdot 1-4$.
}

current, assuming confinement enhancement factors of about 3.0 over L-mode, and standard divertor opcration. The tokamak has port provisions to increase the power in each of the baseline systems to the levels indicated in the "Maximum" column of Table I. This staged approach to implementing the heating and current drive system affords sufficient capability to meet initial objectives while allowing hardware commitments to be guided by results on other machines and by initial TPX operating experience.

Neutral beams will provide efficient current drive as well as ion heating, core fueling, a source of toroidal momentum, and a particle source for key diagnostics. A tangential neutral beam line will be installed initially to provide $8 \mathrm{MW}$ in deuterium at a maximum energy of $120 \mathrm{keV}$, using one of the existing TFTR beams, upgraded for $1000 \mathrm{~s}$ operation. The tokamak provides port access for an additional beam injecting in the same direction as the first and a third in the opposite direction.

The Ion Cyclotron Range of Frequencies (ICRF) system provides electron heating and centrally-peaked current drive in the $40-80 \mathrm{MHz}$ range. The initial system will provide $8 \mathrm{MW}$, coupled through a 12 strap antenna array installed in two adjacent ports, in order to provide a satisfactory launch spectrum for current drive. The straps will be protected by an actively-cooled Faraday shield with a low $Z$ coating $\left(B_{4} C\right)$ for impurity control reasons. A third launcher module, contiguous with the first two, could be added to provide an 18 strap array and up to $18 \mathrm{MW}$ of heating power.

The lower hybrid system provides off-axis current profile control, efficient bulk current drive at low temperature, and electron heating. Klystron sources at 3.7 $\mathrm{GHz}$ and a waveguide-grill launcher will provide 1.5 MW to the plasma. The wavenumber spectrum is variable to support flexible off-axis current profile control in a range of scenarios. The launcher is movable over a $5 \mathrm{~cm}$ range in real time to permit dynamic matching to changing plasma conditions. The lower hybrid system could be expanded to $3 \mathrm{MW}$ by installing a larger launcher and adding sources.

An ECH system may be added for startup assist, disruption control, electron heating, and discharge cleaning. Existing or planned $110 \mathrm{GHz}$ systems, if available, are well suited to the $4 \mathrm{~T}$ magnetic field of TPX.

\subsubsection{Reference Operating Points}

In developing TPX operating points at a zeroD level we have generally employed ITER physics 
rules. ${ }^{6}$ However, we have adopted a more accurate bootstrap current model, used MHD $\beta$-limits consistent with kink and ballooning mode calculations for specific operating modes, and assumed confinement enhancement factors $\mathrm{H}$ in excess of 2 where noted. In defining prudent design requirements it is necessary to be simultaneously optimistic and pessimistic: assuming that operation with $H \geq 3$ and $\beta_{N} \approx 5 \%$ might be achieved when defining the maximum capability of the PF system, while assuming that $H \approx 2$ when sizing the "ultimate" current drive and heating system.

Although the TPX is designed to accommodate up to $45 \mathrm{MW}$ of heating and current drive power the baseline operating points presented in this section require only the $17.5 \mathrm{MW}$ in the initial current drive and heating system. Increases in heating power will provide greater current drive flexibility for current profile control and the additional performance needed to explore very high beta operating regimes. Alternatively, the additional power can be used to explore in steady-state more conventional tokamak operating regimes (i.e., $\beta_{N} \approx 3.5$ and $H \approx 2$ ). At the same time the divertor and first wall systems are designed to handle the full 45-MW complement to demonstrate the handling of advanced reactor heat loads as an extension of the TPX mission.

With the baseline system we project (using our standard physics rules) that it will be possible to produce interesting discharges, including maintenance of a steadystate 1-MA hydrogen discharge or a 1.5-MA deuterium discharge with a combination of bootstrap currents and non-inductive current drive. Even with such conservative confinement assumptions (e.g., confinement enhancement factor $H=2$ ), substantial progress on the TPX mission can be made with the initial heating and current drive system. Assuming that we will be able to extend the confinement enhancement seen transiently in present tokamak experiments (c.g., $H \approx 3$ ) to long pulse operation, the elements of the TPX objectives can be met without enhancements in the heating and current drive system as shown in Table II.

$2 M A$ High- $\beta$ Operation. The first column of Table II shows that full current deuterium discharges can be maintained in steady-state if we are able to combine density control with enhanced confinement operation. This projected operating point reaches the first-stable $\beta$ limit at full current ( $2 \mathrm{MA})$ and full magnetic field (4 $\mathrm{T})$. The core plasma parameters of $18 \mathrm{keV}$ at a density of nearly $1 \times 10^{20} \mathrm{~m}^{-3}$ is clearly reactor-relevant (al-

\footnotetext{
${ }^{6}$ N. A. Uckan et al., (1990). ITER Physics Design Guidelines: 1989, ITER Documentation Series No. 10, IAEA, Vienna.
}

Table II. Enhanced Confinement Operation with Initial Current Drive and Heating System

\begin{tabular}{lccc}
\hline Parameter & $\begin{array}{c}2 \mathrm{MA} \\
\text { high- } \beta\end{array}$ & $\begin{array}{c}\text { High } \\
\text { bootstrap }\end{array}$ & $\begin{array}{c}\text { Very } \\
\text { high- } \beta\end{array}$ \\
\hline Toroidal field, $B_{\mathrm{T}}(\mathrm{T})$ & 4.0 & 4.0 & 3.3 \\
Plasma current, $I_{\mathrm{P}}(\mathrm{MA})$ & 2.0 & 1.4 & 1.2 \\
$q_{95}$ & 3.3 & 5.0 & 4.5 \\
H-factor, $\tau_{\mathrm{E}} / \tau_{\mathrm{ITERg9-P}}$ & 3.0 & 3.0 & 3.0 \\
$\beta_{\mathrm{N}}(\%)$ & 3.1 & 3.3 & 4.0 \\
$\beta_{\mathrm{P}}$ & 0.35 & 0.56 & 0.62 \\
Central density, $n_{\mathrm{co}}\left(m^{-3}\right)$ & $0.94 \times 10^{20}$ & $1.3 \times 10^{20}$ & $1.0 \times 10^{20}$ \\
Electron temperature, $\mathrm{T}_{\mathrm{co}}(\mathrm{keV})$ & 18.3 & 9.4 & 9.9 \\
Ion temperature, $T_{\mathrm{lo}}(\mathrm{keV})$ & 18.9 & 9.4 & 9.7 \\
Energy confinement time $(\mathrm{ms})$ & 322 & 233 & 208 \\
$\tau_{\mathrm{se}} / \tau_{\mathrm{E}}$ & 0.01 & 0.01 & 0.01 \\
Bootstrap fraction, $f_{\mathrm{BS}}$ & 0.42 & 0.72 & 0.54 \\
BS collisionality correction & 0.01 & 0.12 & 0.05 \\
DD neutron rate, $S_{\mathrm{DD}}\left(s^{-1}\right)$ & $3.8 \times 10^{16}$ & $1.8 \times 10^{16}$ & $1.3 \times 10^{16}$ \\
Neutral beam, $P_{\mathrm{NBI}}(\mathrm{MW})$ & 8.0 & 8.0 & 8.0 \\
ICRF, $P_{\text {ICRF }}(\mathrm{MW})$ & 8.0 & 8.0 & 8.0 \\
Lower Hybrid, $P_{\mathrm{LH}}(\mathrm{MW})$ & 1.5 & 1.5 & 1.5 \\
\hline
\end{tabular}

though the thermonuclear performance is not-we project $Q \approx 0.4$ for this discharge).

High Bootstrap Operation. The second column of Table II illustrates operation of a high-bootstrap scenario at a density that should correspond to comfortable divertor operation. This projected operating scenario has $\left\langle n_{e}\right\rangle=0.75 \times 10^{20} \mathrm{~m}^{-3}$, and satisfies the physics constraints for both current profile control $\left(\tau_{\mathrm{se}} / \tau_{\mathrm{E}} \leq 0.12\right)$ and collisionality (collisionality correction $\leq 0.15$ ).

Very High- $\beta$ Operation. The final column of Table II shows a projected operating point in which the first stable $\beta$-limit is substantially exceeded by reducing the magnetic field to $3.3 \mathrm{~T}$. Hence, the initial current drive and heating system will provide sufficient power to test $\beta$-limits at substantial magnetic fields if we can achieve $H \approx 3$ confinement performance.

Hydrogen Operation. During the first 2 years of TPX operation we plan to limit operation in deuterium in order to avoid activating the machine beyond the limit for in-vessel hands-on maintenance. Such discharges will allow us to commission auxiliary systems and diagnostics, verify confinement scaling at high aspect ratio, verify our predictions regarding the scrape-off-layer plasma, begin experiments aimed at developing improved divertors, and develop techniques for active control of the current profile. While hydrogen discharges may not challenge the $\beta$-limit, they do have interesting central temperatures and densities and satisfy both the current 
profile control and collisionality constraints discussed earlier.

\subsubsection{Advanced Operating Modes}

TPX is being designed to explore "Advanced Tokamak" (AT) operating modes with the potential for confinement and beta exceeding contemporary scalings, and bootstrap fraction approaching unity. Six candidate AT modes, distinguished by differences in the plasma current, shape, pressure, and density profiles have been defined to assess operating space, control, and other hardware requirements for AT operation. Equilibrium flux and current profile plots for these modes are shown in Fig. 4.

Four of these six modes are based on operating modes in existing tokamaks, while the modes denoted ARIES-I and ARIES-II have received attention as reactor relevant modes in the ARIES Reactor Design Study where a concerted effort was made to maximize the use of bootstrap current drive. Here, we briefly describe each mode to provide rationale for the choice of the profile functions used.

Standard Tokamak. This is the standard high- $\beta$, highcurrent, first stability regime mode of operation with $q_{0}$ $\approx 1$. The pressure and current profiles here are typical of what a transformer driven tokamak would have.

ARIES-1. This is the mode of operation that was

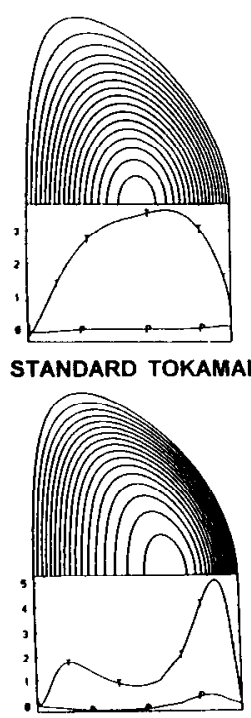

NON-MONOTONIC a
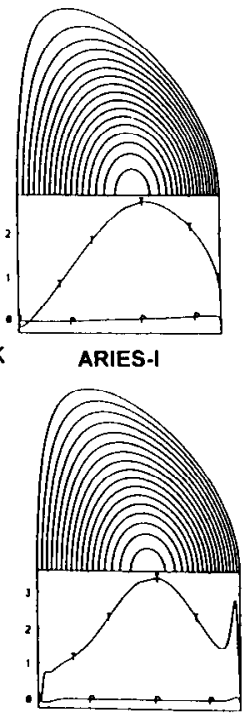

VH-MODE

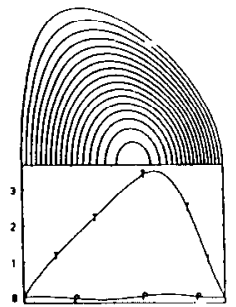

SUPERSHOT

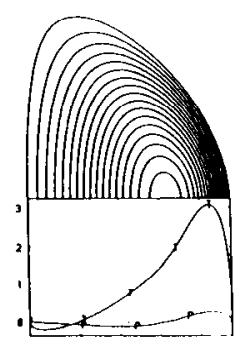

ARIES-II
Fig. 4. Equilibrium flux surfaces and current profiles for advanced operating modes. found in the ARIES-I reactor study to give a good compromise between high- $\beta$ and high- $\beta_{p}$, while remaining in the first stability regime. Its central safety factor, $q_{0}$ has been raised to 1.3 while its total current has been reduced so that $q^{*} / q_{0}$ has increased from 1.8 to 3.0. This mode has both a very high bootstrap fraction for a first stability regime tokamak and a good alignment of the bootstrap current with the plasma current. Several machines have achieved high bootstrap fraction, including TFTR, JET, and JT-60U.

Supershot. This is a first stability regime discharge with peaked pressure and current profiles and with reduced plasma current and elongation. For these peaked profiles, which are characteristic of the enhanced confinement supershot mode of operation in TFTR, we find that instability limits the maximum attainable beta values to $\beta_{N}=1.9$. Supershot operation on TFTR occurs when the limiters are prepared to ensure low particle recycling at the plasma cdge. In TPX, such control will be provided by active divertor pumping instead. Confinement enhancement factors of up to 3.5 have been obtained in TFTR in this operating mode.

Non-Monotonic $q$. This mode seeks to maximize $\beta$ by customizing the pressure and current profiles to allow stable high- $\beta$ peaked pressure profiles. This is accomplished by distributing the plasma current in such a way that the q-profile has reversed shear, $d q / d \psi<0$, in a large central region of the discharge. This reversed shear region permits the central part of the discharge to be in the second stable region, allowing the pressure gradients near the center to become very large while remaining stable to ballooning modes. This mode is an extension (in terms of the size of the reversed-shear region) of high- $\beta$ discharges in DIII-D and JET. In DIII-D, this mode was associated with ellipticity ramping experiments in which the central $\beta(0)$ reached $44 \%$. In JET this mode was obtained using pellet injection and ICRF heating and is called the "Pellet Enhanced Performance" (PEP) mode. It yielded a five-fold increase in fusion reactivity as compared to equivalent $\mathrm{H}$-mode plasmas. We find the combination of off-axis current peaking and high beta allows for a very good match of the bootstrap and the equilibrium current profiles.

$V H$. This mode is an attempt to reproduce the current and pressure profiles typical of the high-confinement VH mode discharges in DIII-D, which are characterized by their low edge shear and low edge collisionality. The pressure gradient is relatively large at the plasma edge, causing a significant bootstrap current to form there. We account for this effect in our equilibrium modelling by adding a "bump" in the current density near the edge. This bump in the current effectively 
limits the maximum achievable $\beta$ in this mode by destabilizing the kink instability. The VH-mode has only been observed on DIII-D with double-null divertors and has also required the use of field error compensation coils.

ARIES-II. The ARIES-II configuration is able to exceed the first stability limit to ballooning modes by the fact that it has a sufficiently elevated central safety factor $q_{0}>2$, and sufficiently peaked current and pressure profiles that it is in the second region of stability. We find that for peaked current profiles there exists a value of $q_{0}$ which allows access to the second stable region for ballooning modes. On the other hand, as the current profile is broadened the value of $q_{0}$ needed for access to, or even for the existence of, a second stable region increases. We find that the primary benefit of this second stability mode is not to achieve higher values of the plasma $\beta$ than can be achieved in first stability. Rather, the benefit is to enable reasonably high values of $\beta$ to be achieved while satisfying the high bootstrap current condition $\epsilon \beta_{p} \geq 1$.

We have analyzed the equilibria corresponding to the six TPX operating modes to determine their stability with respect to ballooning modes and the external kink mode. We expect the experimental beta limit for these modes to be the minimum of the $\beta_{N}$ for high- $n$ and the $\beta_{N}$ for the $n=1$ mode with the wall at $b / a=1.3$, as shown in Table III. With the exception of ARIES-II, all the modes are found to be stable to the kink if we assume that the vacuum vessel and the passive stabilizing plates effectively appear as a conforming conducting shell with a separation distance of $0.3 a$ from the plasma-vacuum interface. This relies on the fact that there is best agreement between experiment and theory when the effects of a conducting wall are included in stability calculations.

\subsection{Reference Plasma and Machine Geometry}

The vacuum vessel geometry and the arrangement of plasma-facing components are based on a reference plasma equilibrium. The key features are shown in an elevation view of the machine in Fig. 5. The plasma current, toroidal field, and shape parameters of this design-basis equilibrium are as specified in Table I. The plasma space envelope is defined by a nominal-width scrape-off layer surrounding the separatrix and intersecting only the divertor targets, ensuring unobstructed transport of heat and particles to the divertor. The plasma and the vacuum vessel interior are up-down symmetric.

The high-heat-flux region of the outboard divertor target is a cylindrical surface about $0.40 \mathrm{~m}$ in height. It defines one wall of a long "slot" that will contain a radiative or gaseous divertor target. The simplicity of a cylindrical surface facilitates the design of a brazed highheat-flux target structure. The inboard target is similarly designed, however the configuration of the inboard divertor is an open one. In the private-flux region of the divertor there is a moderate-heat-flux baffle with sides parallel to, and spaced about $2 \mathrm{~cm}$ from, the separatrices. The exact width of the gaps between the baffle and the target plates are determined by pumping considerations.

On the outboard side of the main plasma, poloidal

Table III. Comparison of Critical $\beta_{N}$ Values set by Ballooning Modes and by Kink Modes for Conducting Wall at $1.3 a$

\begin{tabular}{lccccccc}
\hline Mode & $I_{\mathrm{p}}$ & $q^{*}$ & $\beta^{*}$ & $\beta_{\mathrm{p}}$ & $f_{\mathrm{BS}}$ & $\begin{array}{c}\text { Limiting } \beta_{N} \\
\text { (high- } n \text { ) }\end{array}$ & $\begin{array}{c}\text { Limiting } \beta_{N} \\
(n=1 \text {, with wall } \\
\text { at } b / a=1.3)\end{array}$ \\
\hline 1. Standard & & & & & & & \\
$\quad$ Tokamak & 2.0 & 2.3 & 4.3 & 1.7 & 0.39 & 4.0 & $5.0^{a}$ \\
2. ARIES-I & 1.2 & 3.9 & 2.5 & 2.7 & 0.66 & 3.5 & $4.5^{a}$ \\
3. Supershot & 1.3 & 2.9 & 1.9 & 1.2 & 0.43 & 1.9 & $2.3^{a}$ \\
4. Non-montonic $q$ & 2.0 & 2.4 & 6.3 & 2.5 & 0.93 & 5.5 & 5.5 \\
5. VH & 1.7 & 2.8 & 2.5 & 1.6 & 0.36 & 2.5 & $5.0^{a}$ \\
6. ARIES-II & 0.9 & 5.2 & 3.2 & 6.0 & 1.04 & $5.1^{b}$ & $3.0^{c}$ \\
\hline
\end{tabular}

a These are still high-n unstable in presence of wall.

- $\beta_{N}$ can be increased to the equilibrium limit, although it passes through a high- $n$ unstable region from $\beta_{N}=3.5$ to $\beta_{N}=4.8$.

c Wall is required at $b / a=1.22$ to stabilize $n=1$ at $\beta_{N}=5.1$. 


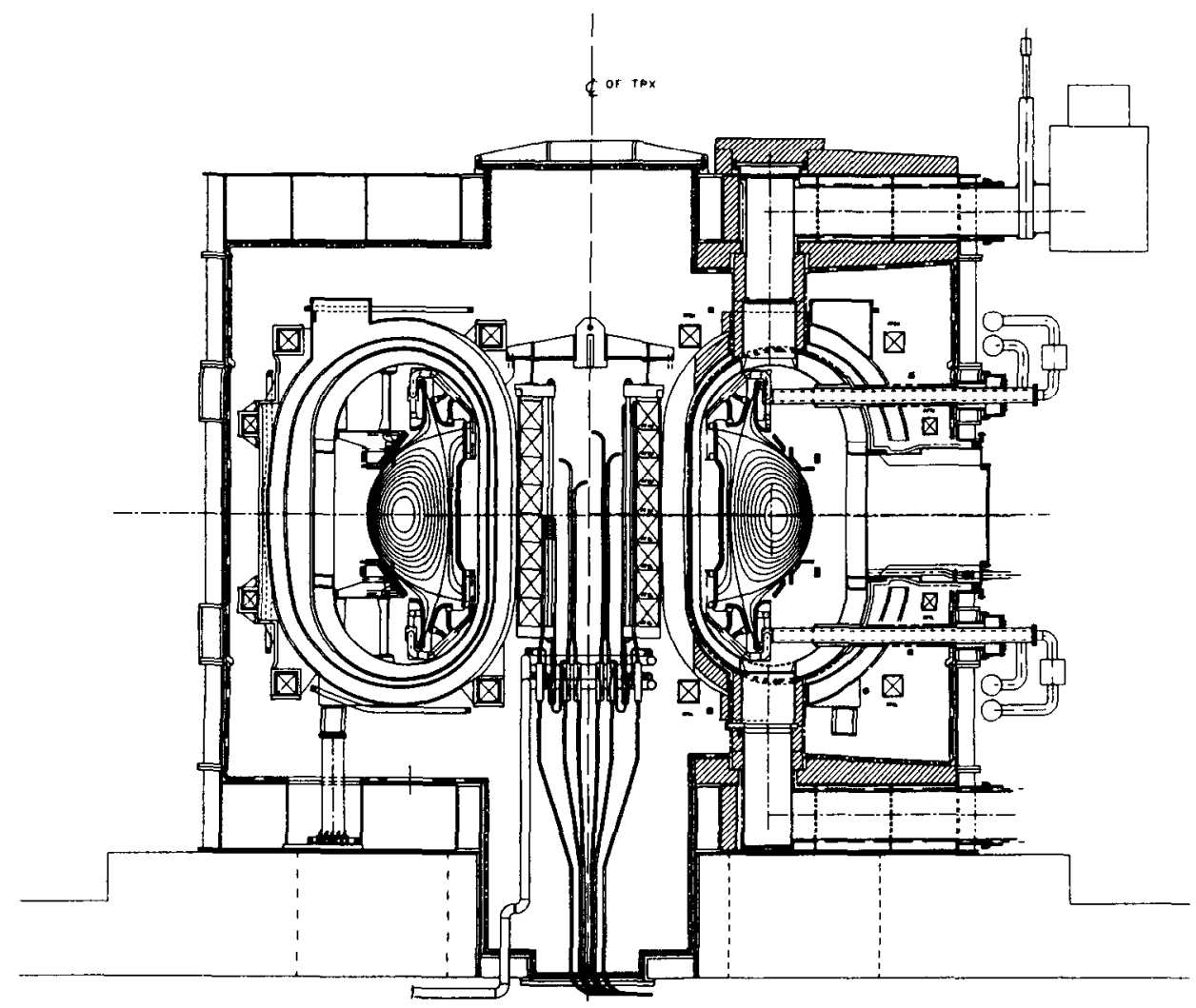

Fig. 5. TPX elevation view.

limiters extend from the midplane to about $0.5 \mathrm{~m}$ above and below it, and conform to the nominal scrape-off surface. These protect the ICRF and lower hybrid launchers installed in the large radial ports and absorb transient heat loads due to startup. The gap between the end of the ICRF launcher and the outer divertor target is spanned by a multi-purpose structure placed tangent to the nominal scrape-off surface. This structure combines a toroidal limiter surface to absorb heat loads due to radiation, ripple-trapped particles, and startup; a support rail for the in-vessel remote maintenance apparatus; and a thick copper conductor which acts as a passive stabilizer, needed to slow the vertical instability growth rate. The conductor is placed as close to the plasma as possible, and spans the maximum poloidal extent possible, in order to satisfy design criteria for passive stabilization of the vertical instability over the largest possible operating space. The upper and lower conductors are connected by a pair of vertical conductors behind one of the poloidal limiters to form a saddle coil that can support up-down antisymmetric eddy currents without in- hibiting the application of loop voltages for startup. The conductor is electrically connected to the vacuum vessel via its titanium supports to avoid high-voltage breakdown problems.

On the inboard side of the plasma, a continuous toroidal belt limiter extends from the midplane to about $0.35 \mathrm{~m}$ above and below it, to absorb heat loads from radiation, neutral beam shinethrough, and plasma startup. Between this limiter and the inner divertor target lies a toroidal limiter-passive conductor structure with a design and purposes similar to its outboard counterpart (except for the remote maintenance aspect).

\subsection{Reference Discharge Scenarios}

Although the TPX is fundamentally designed for steady state operation, transients associated with discharge initiation, current rampup, plasma heating, normal shutdown, and disruptions are important design drivers. The poloidal field (PF) system supports a con- 
ventional inductive plasma initiation with a loop voltage up to $20 \mathrm{~V}$. It provides about 18 volt-seconds to inductively raise the current to $2 \mathrm{MA}$ with ohmic heating only, and to then sustain an inductive flattop for $\sim 10$ seconds. Finally it supplies the equilibrium fields needed to provide the operating space in internal inductance $\left(l_{i}\right)$, normalized beta $\left(\beta_{N}\right)$, and safety factor $\left(q_{95}\right)$ that is needed to support a wide range of advanced-tokamak (AT) modes. Reference discharge scenarios have been developed as a mechanism for establishing design requirements.

\subsubsection{Startup}

In the reference startup scenario, the PF coils are pre-energized to provide an initial poloidal flux bias and a field null in the vacuum chamber at the time of breakdown. The coils are then partially discharged through dump resistors to produce the required $20 \mathrm{~V}$ loop voltage in the vacuum chamber. This ensures the ability to initiate the discharge under relatively dirty vacuum and wall conditions. The initial PF currents can be adjusted to produce the required field null characteristics at the time of breakdown. The pre-programmed poloidal field distribution evolves during the initial current rise to maintain a stable equilibrium until the current reaches $100 \mathrm{kA}$, when feedback control is established. Further startup flexibility can be obtained if necessary through upgrades such as programmable dump resistors or electron cyclotron heating.

\subsubsection{Discharge Evolution}

The primary design basis double-null scenario for the discharge evolution after startup features an inductive rampup to the maximum current ( $2 \mathrm{MA}$ ), heating to the Troyon beta limit $\left(\beta_{N}=3.5\right)$, and an inductively-driven flattop phase to permit a transition to non-inductive current drive. The poloidal field coil current requirements for this HC (for "High Current") scenario are derived from calculated free-boundary equilibria at threc flux states: start of flattop (SOF), start of beta (SOB), and end of beta (EOB). Parameters for these states are listed in Table IV. The SOF values for $\beta_{N}$, internal inductance [denoted $\mathrm{l}_{i}(3)$ ], and flux consumption are based on timedependent simulations of profile evolution in the ohmic rampup phase using the Tokamak Simulation Code. During the current flattop, the PF system provides an additional 0.4 volt-seconds to offset a small increase in the plasma inductance as the beta is raised, plus the required 2.0 volt-seconds consumed resistively.
Table IV. Flux States for Reference HC Scenario

\begin{tabular}{lrrr}
\hline & SOF & \multicolumn{1}{c}{ SOB } & \multicolumn{1}{c}{ EOB } \\
\hline$I_{\mathrm{P}}$ (MA) & 2.0 & 2.0 & 2.0 \\
$\beta_{N}(\%-\mathrm{m}-\mathrm{T} / \mathrm{M} \Lambda)$ & 0.2 & 3.5 & 3.5 \\
$\mathrm{l}_{\mathrm{i}}(3)$ & 0.7 & 0.7 & 0.7 \\
Elapased time (sec) & 4.0 & 5.0 & 14.0 \\
Accumulated flux change (V-s) & -14.8 & -15.4 & -17.2 \\
\hline
\end{tabular}

The 10 second flattop duration in the $\mathrm{HC}$ scenario is set by arbitrarily assuming a resistive loop voltage of $0.2 \mathrm{~V}$. The exact pulse length for this scenario is not critical since the limiting requirement on the PF system is that it be able to maintain continuously any condition bounded by the SOF and EOB states.

The HC scenario alone does not guarantee the capability to run lower-current discharges, because some of the flattop PF coil currents may actually increase or change polarity. In addition, the ability to raise the current to $2 \mathrm{MA}$ from a lower plateau value is needed, for example, to freeze in a high- $\beta_{p}$ profile at low stored energy and maintain it while increasing to high current. A reference double-null Low Current (LC) scenario is defined, therefore, to ensure such capability as well as to provide a wide range of steady-state operating currents (0.4-2.0 MA) with a common startup scenario.

\subsubsection{Configuration Flexibility}

Exploration of advanced tokamak operating modes clearly requires a high degree of flexibility in the range of equilibria that can be accommodated. The advanced tokamak operating modes that have been analyzed from an MHD point of view for TPX define the boundaries of the required operating envelope in safety factor-internal inductance $\left(q_{95}-1_{i}\right)$ space. Based on the operational range of DIII-D it appears that the interesting $l_{i}$ range extends from $\mathrm{l}_{i}(3)=0.5$ at all $q_{95}$ values. However a wider range of $\mathrm{l}_{i}$ is of interest at $q_{95}=5\left[\mathrm{l}_{i}(3)\right.$ up to 1.5 at maximum elongation and even higher at lower elongation] than at $q_{95} \approx 3\left[\mathrm{l}_{t}(3)\right.$ up to 1.2$]$. In DIII-D it is found that operating modes with $\beta$ values exceeding the Troyon limit are contained within the $q_{95}-1_{i}$ operating space just defined. Consistent with the mission of TPX, its $\mathrm{PF}$ system is also required to accommodate high normalized $\beta$ values: $\beta_{N} \leq 5$ at $q_{95} \approx 3$ and $\beta_{N} \leq 7$ at $q_{95}=5$.

While hardware component placement is determined on the basis of a single reference equilibrium, the flexibility to accommodate a range of plasma shapes and 
profiles is retained. The reference geometry defines a set of spatial control points. Plasmas at values of $\beta, 1_{i}, q$, other than those of the reference equilibrium are constrained by these control points. This ensures the proper interface between the plasma and internal components over a wide operating window. An additional constraint on the flexibility is that the passive structure is inadequate to satisfy design criteria for passive stabilization of the vertical instability at low values of both $\beta$ and $l_{i}$. Since this regime is of limited interest, it is excluded from the required operating space for the PF coils, depicted in Fig. 6. The notation $q_{95}=3^{*}$ means that $q_{95}=3$, except where it must be increased to limit $I_{p}$ to 2 MA. To reach those configurations that require slightly more volt-seconds than the reference HC scenario, it may be necessary to rely to a small degree on rampup by noninductive current drive.

In addition to the reference double null scenarios and their flexibility variants, the PF coils must support the equilibrium fields needed for single-null operation at $q_{95}=3^{*}$ over the same range specified for double-null configurations. Special control-point constraints are defined for single-null equilibria. The active null may be either on the top or the bottom.

It should be noted that while the power supply capability to produce the reference $\mathrm{HC}$ scenario is required in the baseline, some upgrades will be necessary to cover the full flexibility range of the coils as described in this subsection. The power supplies will initially be configured for essentially up-down symmetric operation, so only double-null configurations will be available at that time. The requirement for the baseline power supplies to support $2 \mathrm{MA}$ operation (with less than full flexibility) ensures that there will be considerable flexibility to support a wide range of advanced tokamak modes at lower currents.

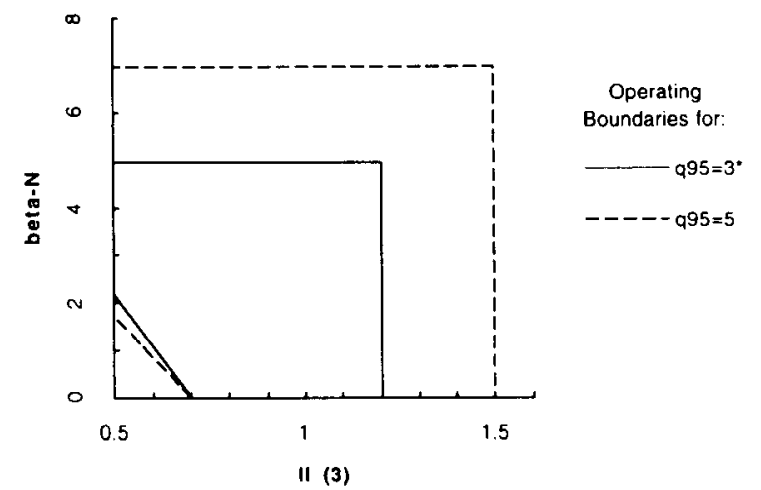

Fig. 6. Requircd cquilibrium opcrating boundaries in $l_{i}(3)-\beta_{N}$ spacc.

\subsubsection{Disruption Evolution}

While developing techniques for disruption control is an important element of the TPX mission, the machine is nevertheless designed to withstand full-current disruptions with a current quench time of $4 \mathrm{~ms}$ or greater. The reference disruption scenarios are specified on the basis of extensive surveys of the experimental database performed under the BPX and ITER CDA design studies. Design scenarios for disruptions include those with a long vertical drift phase prior to disruption (VDEs), and with halo currents up to $25 \%$ of the initial toroidal current. A model for disruptions with halo currents has been implemented in the TSC code and recently benchmarked against DIII-D data. Engineering loads are generated using a 3-D electromagnetics code, SPARK, that models the structure in detail, but uses a simple model for the plasma evolution. The SPARK code does not treat the plasma dynamics self-consistently but prescribes the plasma behavior based on TSC simulations which do so. The capability provided by SPARK is essential for determining loads on non-axisymmetric structures such as the vacuum vessel (with its many appendages), the passive stabilizers, and the RF launchers.

\subsection{Plasma Control}

\subsubsection{Shape and Position Control}

It is clear from the previous sections that careful control of the plasma shape and position is essential to the successful operation of TPX. The nominal gap between the edge of the plasma and components on the outboard side is only $2 \mathrm{~cm}$, and the plasma shape must adjust to changing equilibrium conditions in order to interface properly with the divertor. The system is therefore required to maintain control of the plasma's radial position, the vertical position, and the outer strike point within $\pm 1 \mathrm{~cm}$ of their nominal positions. Magnetic diagnostics, consisting of a toroidal flux loop and a pair of poloidal field pickup probes at each of $\sim 50$ poloidally distributed locations, will be used for this purpose. Conventional magnetic loops combined with low-drift integrators are expected to be sufficient for pulse lengths up to $1000 \mathrm{~s}$. Non-inductive methods such as Hall probes and vibrating probes can be used for longer pulses, but require development.

An important component of the overall plasma control strategy is the control of the vertical instability. Since the TPX mission requires stable operation in regimes of 
high elongation over a wide range of plasma current profiles, it is important to make the $n=0$ stable operating space as large as possible. The two key design features related to this are passive structure and active control coils. The role of the passive structure is to reduce the vertical instability growth rate so as to make active control feasible. Since the vacuum vessel is insufficient for this purpose, toroidal conductors close to the plasma are provided on both the inboard and outboard side, as was discussed earlier. The key parameter for passive stabilization is a function of the passive structure geometry and plasma profiles, especially the current profile as measured by $l_{i}$. In order to satisfy the design criterion over the widest possible operating space, the stabilizers have been designed to be as close to the plasma as possible (making necessary allowance for plasma-facing armor), and to span as large a poloidal angle as possible. Even so, it is not possible to meet the criterion at simultaneously low values of both $\beta$ and $\mathrm{l}_{i}$, so a "corner" of the flexibility space is excluded, as was discussed in the previous subsection.

Active vertical control on fast time scales is provided by a pair of in-vessel coils located between the passive structure and the vacuum vessel. The in-vessel location is necessary to prevent cold-structure inductive heating by the fluctuating control currents. Like the passive structure, these coils are connected in a saddle configuration to facilitate loop voltage penetration during startup and to avoid high induced voltages during disruptions. The operating voltage and current requirements are determined by numerically simulating a feedback control loop based on the plasma's vertical position, assumed to be measured exactly, and a power supply with a fast response time $(<1 \mathrm{~ms})$. Proportional and derivative gains on the position signal are first determined by considering a $2 \mathrm{~cm}$ step change in the vertical position. The optimum gains are chosen based on several factors, including peak current and voltage, response time, settling time, and overshoot. A more realistic scenario is then developed for purposes of power supply design and eddy current heating analysis. In this scenario the plasma position is assumed to fluctuate randomly with an rms amplitude of $1 \mathrm{~cm}$ and a noise power spectral bandwidth $(\Delta \omega)$, equal to the maximum vertical instability growth rate, about $40 \mathrm{sec}^{-1}$.

Other plasma control functions are accomplished with the external PF coils on time scales typical for the standard discharge scenarios (4 sec for current ramp, 1 $\sec$ for $\beta$ rise). The radial position of the outer plasma separatrix is variable over a $\pm 2 \mathrm{~cm}$ range to maintain good impedance matching between the rf launchers and the plasma. The vertical position can be varied over a $\pm 3 \mathrm{~cm}$ range (in addition to the fast control function described above) to compensate for stray magnetic field and possible up-down asymmetries in the system, and to provide a means of balancing the heat and particle loads to the upper and lower divertors. The outer divertor strike point can be varied over a $\pm 5 \mathrm{~cm}$ range to control the interface between the divertor plasma and the pumping plenum.

\subsubsection{Field Error Control}

Control of locked modes is a key element of the disruption control strategy for TPX, so control of critical field error harmonics is necessary. This will be implemented partly through careful design of coil feeds, crossovers, and magnctic structures in the system. It will be an important factor in the establishment of manufacturing and assembly tolerances for coils. However, it is assumed that a system for field error compensation will be necessary for fine-tuning. A basic version of such a system has been successful in controlling the thresholds for locked-mode disruptions on DIII-D. ${ }^{7}$ The TPX incorporates a more comprehensive system of modular field error compensation coils based on a design that has been developed for DIII-D, though not yet implemented. The coils are designed to reduce the normalized amplitude of the helical component of the static error field with poloidal mode number $m=2$ and toroidal mode number $n=1, \delta B_{r(2,1)} / B_{\mathrm{r}}$, to a low value: $<1 \times 10^{-4}$. The compensation coils are configured to produce a harmonic spectrum similar to that of shifted or tilted PF coils. Preliminary current requirements for the compensation coils have been determined assuming typical PF coil displacements. However the engineering tolerances for coil positioning accuracy at operating temperatures have not yet been established.

\subsubsection{Ripple}

Limiting the toroidal field ripple is an important design consideration, especially in a steady-state device. Energetic particle losses due to ripple trapping tend to concentrate in local "hot spots" on the outboard side of the plasma, potentially resulting in high peak heat loads. This imposes a more restrictive limit than plasma power balance considerations. The maximum allowable ripple

${ }^{7}$ R. J. LaHaye, A. W. Hyatt, and J. T. Scoville (1992). Non-Linear Instability to Low $m, n=1$ Error Fields in DIII-D as a Function of Plasma Fluid Rotation and Beta. General Atomics Report GA-A20824, May. To be published in Nucl. Fusion. 
in TPX is kept quite low for this reason, less than $0.4 \%$ over the plasma cross-scetion. The main concern is energetic neutral beam ions, since minority ion cyclotron heating is not a primary operating scenario.

Based on Fokker-Planck code analysis, energy losses due to ripple trapping is less than $5 \%$ and the attendant peak heat loads on outboard components are estimated to be about $1.5 \mathrm{MW} / \mathrm{m}^{2}$.

\subsection{Power and Particle Handling}

The design of power and particle handling systems is driven equally by the steady-state and the advancedtokamak aspects of the TPX mission. Active cooling is required to handle high steady state heat loads and active pumping is needed to exhaust particles. The configuration is designed to support advanced divertor operating modes, using a radiative gaseous target to more uniformly distribute the heat loads onto material target surfaces. It must also maintain a high recycling divertor plasma consistent with $\mathrm{H}$-mode requirements, while exhausting a small fraction of the recycling particles to maintain global particle balance. Materials choices are also influenced by multiple considerations: plasma performance, component lifetime, and low activation.

\subsubsection{Maximum Heating Power}

The plasma-facing components are designed to handle a maximum steady state heating power of $45 \mathrm{MW}$, in order to accommodate possible heating upgrades. This corresponds to an average heat flux across the separatrix of $0.75 \mathrm{MW} / \mathrm{m}^{2}$, which is what must be handled in an attractive DEMO operating at a neutron wall load of 3 $\mathrm{MW} / \mathrm{m}^{2}$. Heat loads to individual components are determined by partitioning the $45 \mathrm{MW}$ among various loss channels. Predictions of peak divertor heat fluxes under advanced steady-state operating conditions are uncertain, however, since it is part of the TPX mission to produce the required data base. The approach being taken is to design the divertor targets to the "technology limit" for power handling, about $15 \mathrm{MW} / \mathrm{m}^{2}$. This will enable them to handle the $18 \mathrm{MW}$ of heating power included in the project baseline, based on standard physics models with conservative safety factors. The experimental data base suggests that standard divertor operation may accommodate even more power than that, but advanced operation is almost certainly required to accommodate the full $45 \mathrm{MW}$. Therefore, the development of advanced divertor operating techniques will be an early objective, in parallel with other objectives, for the TPX experimental program.

\subsubsection{Heat Loads}

The design heat loads to individual components are determined by partitioning the losses from the main plasma (45 MW maximum) among various loss channels for radiation and conduction to the divertors. Neutral beam shinethrough and ripple losses are added in certain localized regions. The partitioning distributions are based on the experimental data base for divertor tokamaks. Two limiting conditions are used to develop the peak heat loads: a high-core-radiation (HR) and a low-coreradiation (LR) condition. In the HR condition, $50 \%$ of the core power is assumed to be lost via radiation; the LR condition assumes $20 \%$ core radiation. The HR condition maximizes the heat loads on components near the midplane, while the LR case maximizes heat loads to the divertor. The power to the divertor targets and baffles is determined based on a $20 \%$ up-down imbalance and a maximum power fractions of $80 \%$ to the outboard target, $50 \%$ to the baffle (consistent with successful radiative divertor operation), and $33 \%$ to the inboard target. Only the outboard divertor target required heat dispersal via advanced divertor operation to handle the full 45 MW of input power.

Neutral beam shinethrough power is partly deposited on the inboard toroidal limiter, and partly in the outer wall region facing the neutral beam ports, where additional armor is required to provide a beam dump. Spccial power handling surfaces are also required on the midplane end of the passive stabilizers to handle ripple losses, and on the outer wall to handle core radiation losses.

\subsubsection{Fueling and Particle Handling}

The maximum main-plasma fueling requirement $\left(5 \times 10^{21} \mathrm{~s}^{-1}\right.$, or 85 torr-1/s), is based on an assumed volume-averaged density and a conservative estimate of the global particle confinement time. Neutral beams will provide about $0.9 \times 10^{21} \mathrm{~s}^{-1}$ per $8 \mathrm{MW}$ and the remainder will be provided by gas puffing and, in the future, a pellet injector and additional beam lines. The divertor pumping system is required to exhaust the maximum main-plasma particle losses at a pressure of $1 \mathrm{mtorr}$, which corresponds to standard divertor operation. The required pumping speed is thus 85,000 torr- $1 / \mathrm{s}$, achieved with a system of external cryopumps connected to the 
divertor region through a total of 16 large-diameter ducts. The volume of the cryopump tanks is determined by the requirement to handle $10^{6}$ torr-I while remaining below the explosive concentration of hydrogen; this accommodates operation 85 torr-1/s for a standard operating day $(10,000 \mathrm{~s})$ without regeneration of the pumps.

Gas target data from DIII- $D^{8}$ suggests that raising the pressure to 3 mtorr could lead to a five-fold reduction in peak heat fluxes. Gas injectors in midplane and divertor region are required to provide flexibility in supplying additional fuel to the scrape-off plasma if needed to produce such condition. The peak throughput requirement is therefore 250 torr- $1 / \mathrm{s}$, consistent with plenum pressures up to 3 mtorr at full pumping speed. For plenum pressures up to $10 \mathrm{mtorr}$, a variable aperture is installed in the pump ducts to reduce the pumping speed. The main baffle in the private region of the divertor is designed to minimize gas leakage from the plenum back to the divertor chamber, and tight-fitting auxiliary baffles between the divertor targets and the vessel wall are installed to reduce the leak rate back to the main chamber to one-tenth of the pumping speed, $8500 \mathrm{l} / \mathrm{s}$.

\subsubsection{Plasma-Facing Materials}

Carbon-based materials such as carbon-fiber composites will be used for the baseline plasma-facing components, including the divertor targets, baffles, limiters, and additional armor. Operating experience on most tokamaks in the last 15 years has consistently shown a strong preference for the use of low $Z$ materials for optimum performance. Carbon is chosen over beryllium because of concerns about melting, especially in disruptions. Calculated net erosion rates for carbon divertors in TPX are about $100 \AA / \mathrm{s}$ for high-performance deuterium operation. This translates to acceptable lifetimes for the initial divertor, since much of the operating time will be with hydrogen and at less than maximum performance. In addition, likely variations in the divertor strike point position (where the erosion peaks) will further increase the lifetime by spreading the erosion patterns over a larger area. Finally, carbon is more tolerant of high plasma temperatures than high $Z$ materials like tungsten or molybdenum, which undergo runaway self-sputtering at temperatures above $50 \mathrm{eV}$. At the same time, beryllium may prove to be advantageous for controlling wall inventories, and high $Z$ materials are of considerable

\footnotetext{
${ }^{8}$ T. Petri et al., (1992). Divertor heat flux reduction by $D_{2}$ injection in DIII-D. Bull. Am Phys. Soc. 37, 1565 (abstract).
}

interest for steady-state reactor applications. The TPX divertor is modular and remotely replaceable, so it can accommodate a changeover to these alternative materials at a later time if desired.

\subsubsection{Vacuum and Wall Conditioning}

Vacuum and wall conditions are expected to be quite important for the transient startup phase of TPX discharges, just as they are for pulsed tokamaks with parameters and wall materials similar to those of TPX. In Tore Supra, boronization, baking at high wall temperature, and operation at moderatc wall temperatures are all important ingredients for very long pulse $(60 \mathrm{~s})$ passive limiter operation with noninductive current drive. With pump limiters, Tore Supra has found that the pumps can act directly on the particle reservoir retained in the wall without affecting the plasma population.

The TPX will be designed using high-vacuum techniques and approved materials consistent with maintaining low base partial pressures. To purge impurities after a vacuum opening, the vacuum vessel, horizontal ports, pump ducts, and all in-vessel components will be baked at $350^{\circ} \mathrm{C}$. Operation with hot walls is important for plasma reliability, but is complicated by the high heat removal requirements. Between discharges, the carbon plasmafacing components will be maintained at a temperature of at least $150^{\circ} \mathrm{C}$ using high-temperature water. However, the heat transfer properties of water at this temperature are inadequate to handle the high heat loads of full power divertor operation, so it will be necessary to switch to low-temperature cooling water a few minutes prior to discharge initiation. This will cause the temperature of the carbon surfaces to drop briefly, but they will rise to as high as $1100^{\circ} \mathrm{C}$ under steady state heat loads. Glow discharge cleaning (GDC) and boronization procedures, with the walls at bakeout temperatures, will be employed.

\subsection{Diagnostics and Data Handling}

The TPX will initially be configured with a basic diagnostic complement necessary for machine operations and for evaluation of advanced tokamak operating modes. Major diagnostic categories include those needed for plasma control, core confinement, divertor and edge plasma conditions, fluctuations, and fusion products. The initial set of diagnostics will consist of those listed in 
Table V. In addition, the device has ample port space to accommodate a large number of diagnostic upgrades.

The steady state mission also imposes special requirements on the data handling system. It will provide for real-time display and operator intervention during the course of a discharge, and in addition will permit coordination of operation and data analysis among multiple sites.

\subsection{Operation}

The operating plan for TPX considers the range of operating scenarios necessary to achieve initial objectives, as well as the staging of hardware capabilities such as heating and current drive systems and diagnostics. The baseline facility is designed for $1000 \mathrm{~s}$ deuterium pulses at 75 minute intervals to a maximum of 10 per day. Short-pulse operation at more frequent intervals will be essential for efficient optimization and exploratory studies, so a scenario of $100 \mathrm{~s}$ deuterium pulses at 20 minute intervals to a maximum of 30 per day is also accommodated. Experience on operating tokamaks such as DIII-D suggests that about 100 operating days per year is a reasonable expectation. The baseline TPX facility is designed on the basis of an annual 3000 pulses and $5 \times 10^{5}$ seconds of operation, which allows a flexible mixture of $1000 \mathrm{~s}$ and $100 \mathrm{~s}$ pulses. Lifetime operating limits of 10 years and 30,000 pulses are assumed in the design.

Deuterium operation is constrained by the need to limit radiation dose levels to maintenance personnel. An annual DD neutron budget of $6 \times 10^{21}$ and an annual limit on deuterium operation of $2 \times 10^{5} \mathrm{~s}$ have been adopted. This corresponds to an average neutron rate of
$3 \times 10^{16} \mathrm{~s}^{-1}$, although the shielding and refrigeration are designed for a peak neutron rate of $5 \times 10^{16} \mathrm{~s}^{-1}$ (upgradable to $1.2 \times 10^{17} \mathrm{~s}^{-1}$ ). To optimize operational productivity, maintenance dose levels will be minimized in the initial operation, while in-vessel remote maintenance procedures are being established. Hands-on maintenance of in-vessel components will be possible during this time period. This will be accomplished through the phased implementation of long-pulse deuterium operation and the use of low-activaiton materials (e.g., a titanium vacuum vessel). This approach permits substantial long pulse operation in hydrogen, and sufficient deuterium operation (in terms of performance and pulse length) to explore advanced-tokamak issues such as current profile control, and to evaluate beta limits.

Provision has been made for short-pulse DT operation in TPX at the end of its experimental period. A peak DT neutron source rate of $5.3 \times 10^{18} \mathrm{~s}^{-1}$ (corresponding to $15 \mathrm{MW}$ of fusion power) is specified. The annual yield is expected to be limited to $1 \times 10^{21} \mathrm{DT}$ neutrons, based on the site envelope established for TFTR. Advanced regimes can be set up in long deuterium pulses, and then the plasma composition can be rapidly adjusted to a 50:50 DT mix, to allow a few seconds of operation in the presence of an energetic alpha population. This will permit tests of the robustness of TPX's advanced regimes against alpha-driven instabilities.

The TPX experimental program will have several elements in its initial years: operating limits, plasma control, large-aspect-ratio physics, and divertor physics. Success in these areas will produce the building blocks needed to achieve the TPX mission objectives. At the same time valuable experience will be gained in the operation of an integrated superconducting magnet system and in remote maintenance operations. It is important to

Table V. Initial Diagnostics

\begin{tabular}{lll}
\hline Machine diagnostics & Physics diagnostics & Divertor/edge diagnostics \\
\hline Magnetic loops & Visible filterscopes & Divertor IR TV \\
Hard X-ray detectors & Visible bremsstrahlung & Visible $\mathrm{H}_{\alpha}$ TV \\
Millimeter-wave interferometer & Charge exchange spectroscopy & Foil bolomcters \\
UV survey spectrometer & Thomson scattering & Divertor bolometer arrays \\
Plasma and infrared TV & Motional stark effect (MSE) & Multichord visible spectrometer \\
Visible survey spectrometer & Soft X-ray arrays & Fast ncutral pressurc gauges \\
Residual gas analyzer & Epithermal ncutron detectors & $\mathrm{H}_{\alpha}$ monitors \\
Torus ion gauges & ECE heterodyne radiometer & Thermocouples \\
Glow discharge probes & Bolometer arrays & \\
Inspection illumination system & Fixed edge probes & \\
\hline
\end{tabular}


establish confidence in the in-vessel remote maintenance equipment and procedures in the initial 2-year period of hands-on access.

\subsection{Summary}

The design requirements for TPX define a machine with an impressivc capability. The steady state tokamak based on all-superconducting magnets and actively-cooled plasma-facing components will be a truly unique facility for the development of continuous modes of operation. A plasma configuration has been defined with a shaped cross section consistent with advanced-regime stability, and a high aspect ratio. The major parameters together with the use of deuterium fuel provide more than enough performance to produce a reliable data base for an advanced tokamak demonstration reactor.

Most importantly, the TPX will be equipped with a set of advanced plasma controls. This is essential to make the transition from transient modes of operation controlled by initial conditions to steady state modes where the plasma's confinement, stability, and edge conditions are under control of the operator. The heating and current drive, divertor, and PF coil systems provide the key tools needed to make this critical transition in the way tokamaks are operated.

\section{ENGINEERING DESIGN}

Development of the TPX design was an iterative process which involved:

- defining physics requirements and design criteria,

- developing the tokamak configuration and subsystem designs,

- developing cost estimates and algorithms, and

- exploring design alternatives.

The TPX design evolved out of a national effort to define the features and parameters of the "Post-TFTR Initiative."

Optimization studies led to the following conclusions:

- Good confinement $(H>2)$ and high beta $\left(\beta_{N}>3.5\right)$ are both necessary for an attractive DEMO reactor; therefore TPX must be capable of operation at beta values well beyond the first stability limit $\left(\beta_{N} \geq 5\right)$ and have substantial flexibility in plasma shaping, fueling, current profile control, and in controlling divertor/edge conditions to explore a wide range of confinement regimes.

- Reactor studies suggest that an aspect ratio of between 4 and 5 is attractive for reducing current drive power requirements; 4.5 was selected as the TPX design point.

- A major radius of $2.25 \mathrm{~m}$, a toroidal field of 4 $T$ and 2 MA of plasma current provide sufficient performance margin for advanced tokamak experiments.

- If VH-mode confinement $(H-3)$ can be achieved under steady state conditions, advanced tokamak (high beta, high bootstrap fraction) regimes can be explored with only modest heating/current drive complements; for TPX, the initial heating/current drive complements are $8 \mathrm{MW}$ of ICH/FWCD, $8 \mathrm{MW}$ of NB, and 1.5 MW of LHH/LHCD which are adequate for exploring advanced tokamak regimes with $\mathrm{VH}$-mode confinement.

- In order to ensure high electron temperatures and reduce fast ion contributions to the plasma pressure, provisions to accommodate up to $18 \mathrm{MW}$ of ICH/FWCD and $24 \mathrm{MW}$ of NB ( 3 TFTR beam lines) are included in the facility.

- A high triangularity $\left(\delta_{x}=0.8\right)$, double null divertor was adopted, since theory and most experiments favor such a configuration for advanced tokamak studies. However, single null capability was retained in order to permit SN-DN comparison in advanced regimes.

- A "slot" divertor geometry was adopted to support radiative/gaseous target operation in a configuration that provides good pumping.

TPX machine parameters are presented in Table I. An elevation view of the tokamak is provided in Fig. 5 . The design features sixteen superconducting $T F$ coils and fourteen superconducting PF coils, symmetrically located about the plasma midplane. The eight inner $\mathrm{PF}$ coils form the central solenoid (CS) assembly. A cryostat encloses all of the superconducting coils. The TF assembly and vacuum vessel are assembled in quadrants with four TF coils per quadrant. Each vacuum vessel quadrant features three large horizontal ports for heating and current drive systems, diagnostics, and remote maintenance access. Vertical Ports are provided for vacuum pumping and additional diagnostic access.

TPX will be assembled in the TFTR test cell after decontamination and decommissioning of TFTR has been completed. Figure 7 shows a plan vicw of TPX inside the TFTR test cell (with the maximum complement of NB heating). 


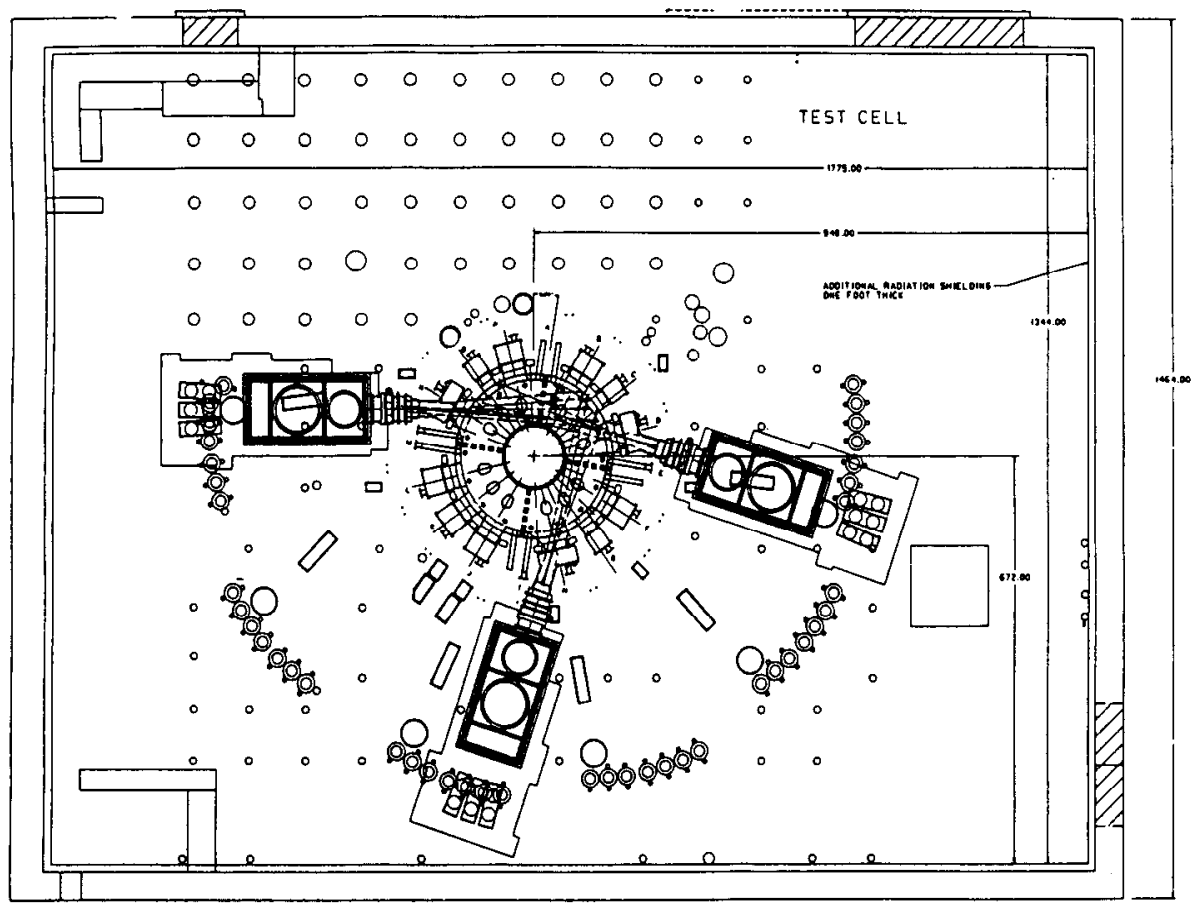

Fig. 7. TPX plan view inside TFTR test cell.

The TFTR facility is well-suited to TPX needs. Many TFTR systems including the power, neutral beam (NB), ion cyclotron (IC), diagnostics, and water systems will be reused. Only two new buildings are required: the LHe Refrigeration Building and a Water Systems Pump Building. Adequate space is available on " $D$ " site at PPPL to expand the heating, power, and water systems if necessary. Figure 8 shows the TPX building arrangement at " $D$ " site.

\subsection{Tokamak Systems}

A toroidal array of sixteen TF coils produce the $4 \mathrm{~T}$ toroidal field. The physics requirement of low ripple (less and $0.4 \%$ within the plasma cross-section) and neutral beam access tangential to the plasma at $2.0 \mathrm{~m}$, were leading factors in determining the size and number of TF coils. The TF system is designed so that the inward magnetic forces are reacted by wedging the nose sections of the $16 \mathrm{TF}$ coils.

The TF coils are assembled in four-coil quadrants as shown in Figure 9. The four-coil quadrant accommodates a vacuum vessel quadrant with three large horizontal ports, a pair of upper and lower divertor pump ducts, a central set of vertical diagnostic ports and hor- izontal auxiliary diagnostic ports located at the ends of the quadrant. The four-coil quadrant is built from a pair of two-coil TF modules. The two-coil TF module assembly consists of a central structural weldment and two end weldments which complete the coil assembly. The conductor winding is inserted into the central weldment from the sides. A final closure weld is made between the central and end assemblies along the inside and outside surfaces. One TF assembly end weldment is an electrically isolated bolted interface that accommodates a full horizontal port; the second weldment provides a final quadrant-to-quadrant closure weld interface. The factory assembled four-coil quadrant reduces the final installation fit-up at the nose of the coil to four $\left(90^{\circ}\right)$ wedged interface surfaces.

The TF and PF conductors are internally-cooled, cabled superconductors (ICCS) based on the US-Developmental Poloidal Coil (DPC) conductor, as shown in Fig. 10. The conductor for the TF coils carries the highest current and has the largest number of superconducting strands. The TF conductors and PF5 employ a single, outer sheath. The other PF coils have a double sheath as in the DPC conductor and have the same total conductor cross-section as the TF and PF5 conductors. The sheath for the conductor is made of Incoloy 908, which was chosen for its mechanical compatibility with the 


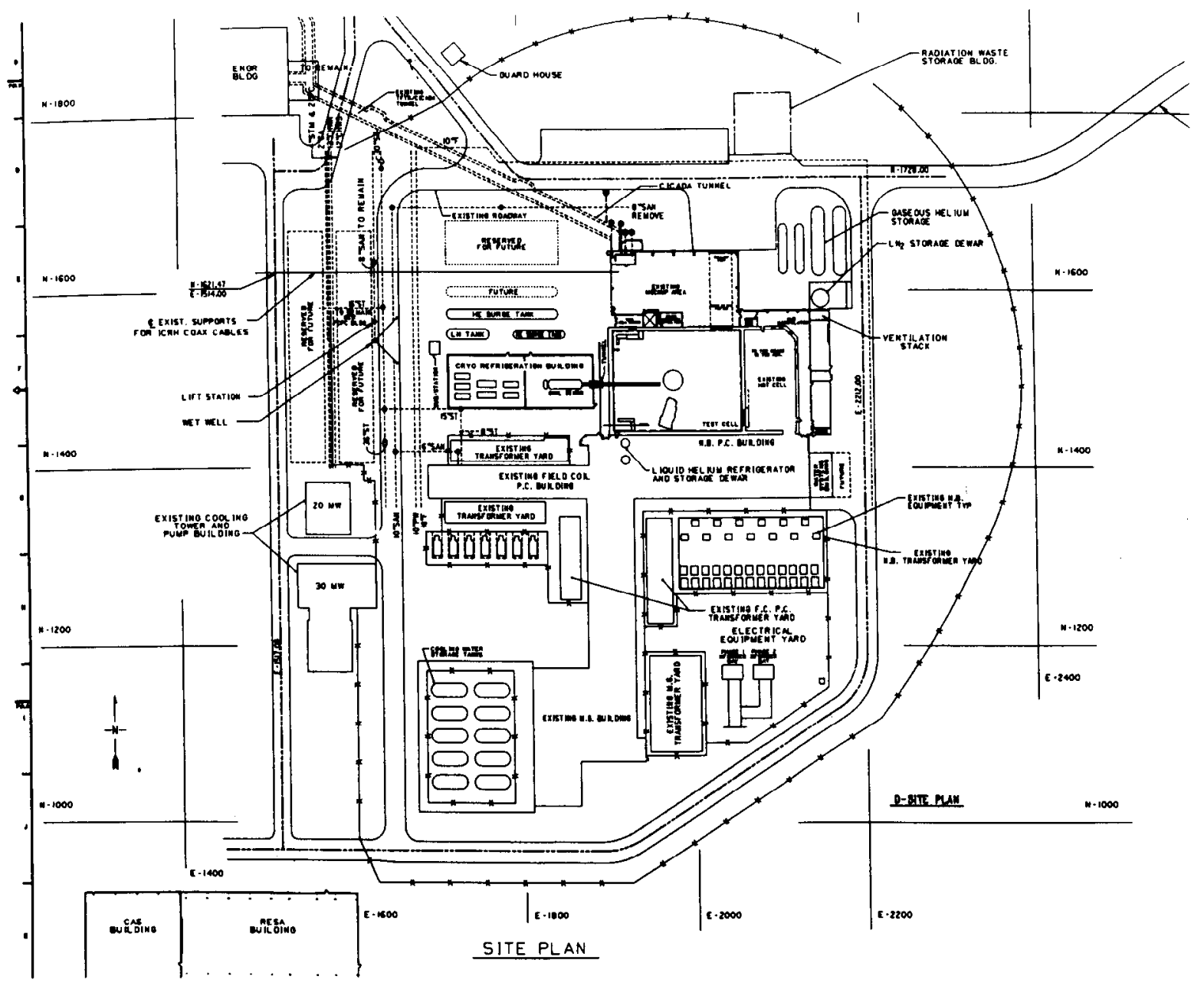

Fig. 8. TPX site plan.

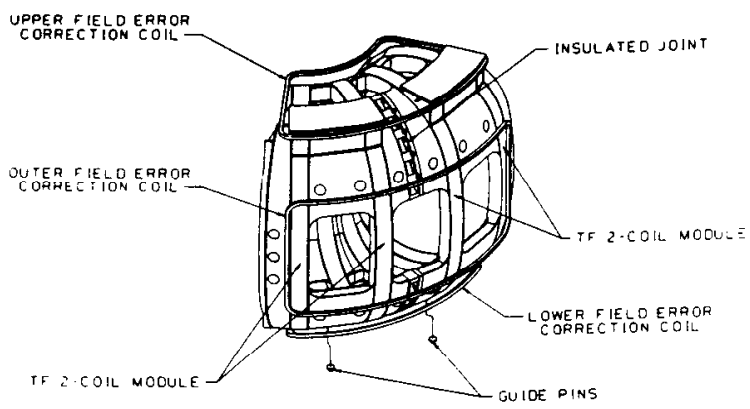

Fig. 9. TF quadrant assembly.

$\mathrm{Nb}_{3} \mathrm{Sn}$ superconductor and its high resistance to cyclic fatigue. The conductor for the TF and PF1 to PF5 is
$\mathrm{Nb}_{3} \mathrm{Sn}$, while the PF6 and PF7 conductor is Nb-Ti. The $\mathrm{Nb}_{3} \mathrm{Sn}$ conductors are reacted at a temperature between $650^{\circ} \mathrm{C}$ and $700^{\circ} \mathrm{C}$ to convert the $\mathrm{Nb}$ and $\mathrm{Sn}$ into the superconducting compound $\mathrm{Nb}_{3} \mathrm{Sn}$.

Each of the $16 \mathrm{TF}$ coils contains 84 turns in the form of 12 pancakes of seven turns each. The coils are wound with a continuous length of conductor to avoid joints (splices) and because of critical space requirements in the region where the helium is supplied to the coil. The TF coil is formed by a multiple-roller winding fixture that bends the conductor and positions it into in its proper final location. After the coil is reacted it is wrapped with glass cloth, insulating strips are inserted and the entire winding pack is epoxy impregnated to form an insulation system capable of withstanding the $15 \mathrm{kV}$ quench protection voltages. Each TF coil is placed in a $316 \mathrm{LN}$ steel case which, along with the intercoil 

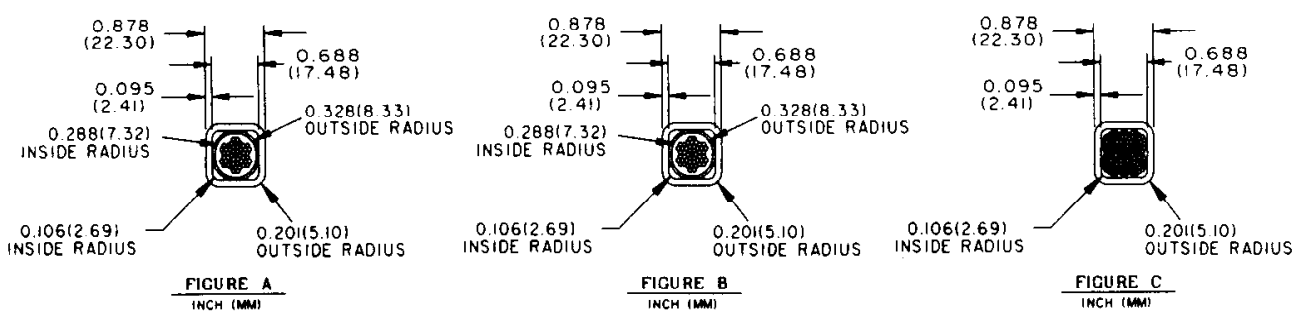

\begin{tabular}{|c|c|c|c|c|c|}
\hline COIL & FIGURE & CONOUCTOR MATERIAL & JACKET MATERIAL & NUMEER OF STRANDS & DIAMETER OF STRANDS IN.(MM) \\
\hline POLOIDAL FIELO I THRU 4 & A & $\mathrm{N}_{3}{ }_{3} \mathrm{Sn}$ & INCOLOY 908 & 225 & $0.031(0.780)$ \\
\hline POLOIDAL FIELD 5 & c & $\mathrm{Nb}_{3} \mathrm{Sn}$ & INCOLOY 908 & 375 & 0.03110 .7801 \\
\hline POLOIDAL FIELD 6 AND 7 & B & NbTi & $316 \mathrm{LN}$ & 225 & $0.031(0.780\}$ \\
\hline TOROIDAL FIELD I THRU 16 & $\mathrm{c}$ & $\mathrm{Nb}_{3} \mathrm{Sn}$ & INCOLOY 908 & 405 & $0.031(0.780)$ \\
\hline
\end{tabular}

\section{TF and PF Coil Conductors}

Fig. 10. TF and PF conductors.

structure withstands the magnetic forces produced by the coils.

All PF coils are circular and are formed with a winding fixture similar to that for the TF coils. They are reacted (for the $\mathrm{Nb}_{3} \mathrm{Sn}$ ), insulated, and cured. To minimize eddy current losses, the PF coils do not have cases. The eight inner PF coils form the central solenoid (CS) assembly, shown in Fig. 11. The CS assembly is attached to the TF coil assembly at the top. A crane pick point is provided to allow the CS assembly to be removed with the overhead crane.

The TF and PF coils are cooled by supercritical helium that is applied at a pressure of 8 atmospheres. There are many separate channels for helium in each coil, and voltage isolation is provided in each separate path. Helium also passes through panels attached to the TF cases in order to intercept nuclear and eddy current heat from the intercoil structure. The TF and PF coils are housed in a large cylindrical cryostat which is evacuated prior to cooling down the superconducting coils to minimize convective heat transfer.

The TF and PF coils will not be geometrically perfect when installed. Even small field errors due to misalignments or eccentricities in the TF and PF coils or other sources (e.g., buswork and magnetic materials) can affect plasma behavior. Field error correction (FEC) coils are provided in order to control the thresholds for locked modes and thereby reduce the incidence of disruptions. The FEC coil system consists of twelve "window frame" shaped coils of conventional copper/glass epoxy insulation design. Three coils are provided on each module: one above, one below, and one mounted on the outer periphery, as shown in Fig. 9. The coils are configured such that they can be mounted on the quadrant during the pre-assembly operations; there are sufficient gaps between the coils to permit the quadrant welding to be performed with them in place. The coils are bussed in like pairs $180^{\circ}$ apart to avoid $n=0$ harmonics and coupling with the axisymmetric PF coils.

The TF assembly is mounted to the tokamak support structure (Fig. 12) which is attached to the cryostat base structure. The tokamak support structure provides support for gravitational loads, seismic loads and electromagnet loads. Provisions are included for differential thermal excursions due to cooldown of the magnets. In addition, a high resistance to heat flow, from the near room temperature conditions at the cryostat base to the $4 \mathrm{~K}$ temperature at the interface with the $\mathrm{TF}$ magnet structure, is provided. The support structure also maintains the concentricity of the machine with respect to its central axis.

The vacuum vessel is a double walled structure located within the bore of the TF coils. It consists of four quadrants which are field welded together at assembly. The vessel material is Titanium $6 \mathrm{AL}-4 \mathrm{~V}$ selected for its relatively low activation compared to other materials, high strength at elevated temperatures, and high electrical resistivity. Ribs serve to attach the inner and outer walls as well as providing rigidity. Double rings above and below the horizontal ports provide additional structural rigidity.

There are twelve large rectangular horizontal ports 


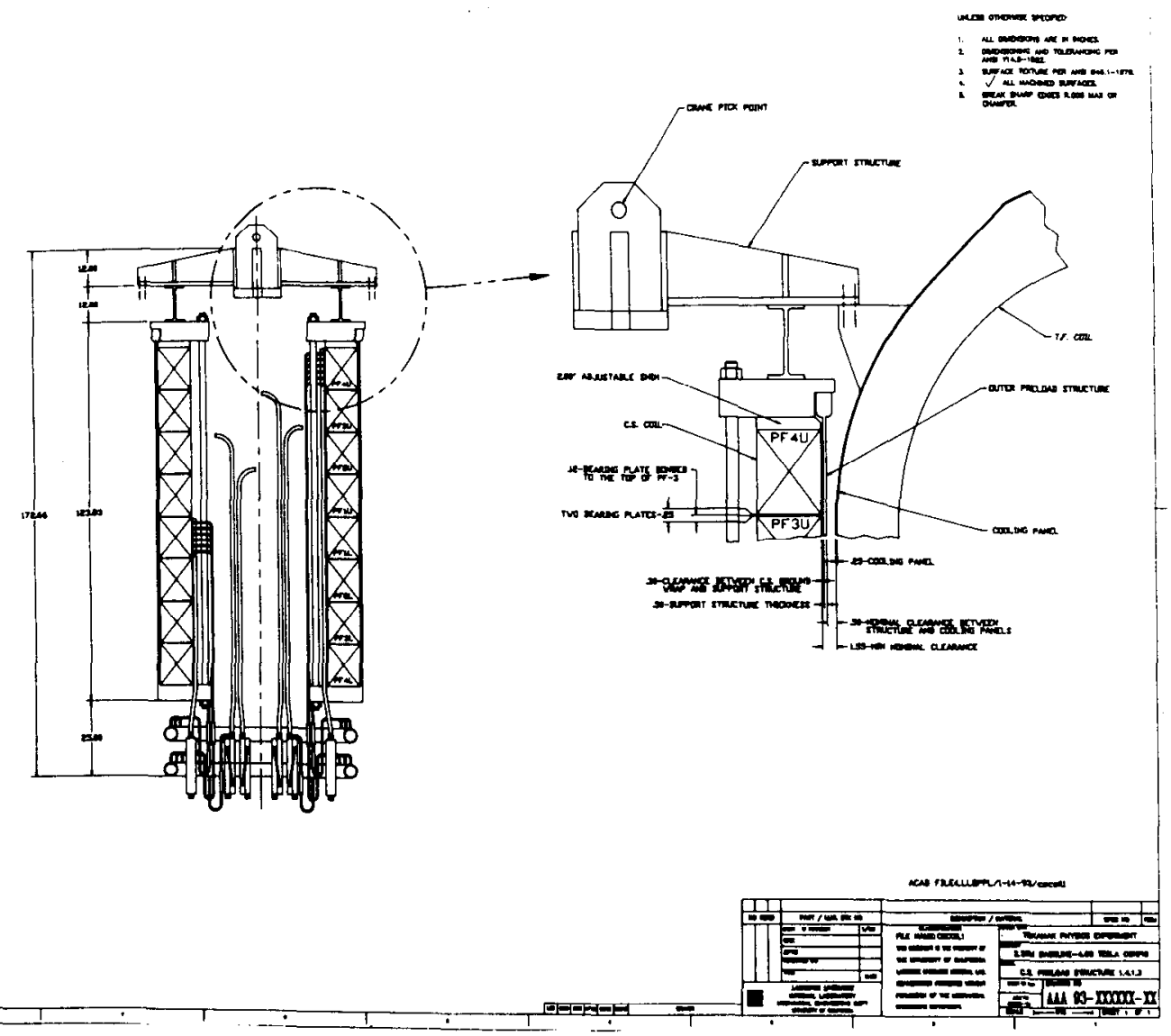

Fig. 11. Central solenoid (CS) assembly.

on the outboard midplane provided for remote maintenance access, diagnostics, and plasma heating and current drive. Twenty-four small circular ports located at the midplane near the assembly joint provide access for services and diagnostics. There are 32 circular ports located above and below the midplane providing the supply/return cooling water for the divertors.

Port allocations are shown in Fig. 13. Of the 12 large horizontal ports, three can accommodate neutral beams. Three large horizontal ports are dedicated for ICH/FWCD. The IC ports are adjacent for optimal current drive. One large horizontal port is dedicated for LHCD. Access for in-vessel remote maintenance is provided through a large port which is shared with diagnostics which are readily demountable. The remaining four large horizontal ports and the majority of the small circular ports at the midplane are dedicated to diagnostics.

Vertical ports are located at the top and bottom of the vacuum vessel with centerlines aligned with the large horizontal ports (12 top- 12 bottom). The vertical ports have a bathtub shape to provide maximum viewing area between the TF coils. Once the port ducts are outside the TF coil envelope a flat transition plate is utilized to make a transition to circular 24 inch pipe. In each quadrant the outer two vertical ports are connected to the vacuum pumping ducts. The middle ports have blank covers which can be used for diagnostic access.

Vertical supports of a pin and link design are provided at the four corners of the lower transition plates that interface with the pumping ports. These supports transmit vertical loads to the cryostat base structure. Radial and tangential supports are provided at each large horizontal port and transfer their loads into the cryostat structure.

All of the large horizontal ports terminate in flanged connections which utilize double metallic seals. The space between the double seals is connected via small diameter 


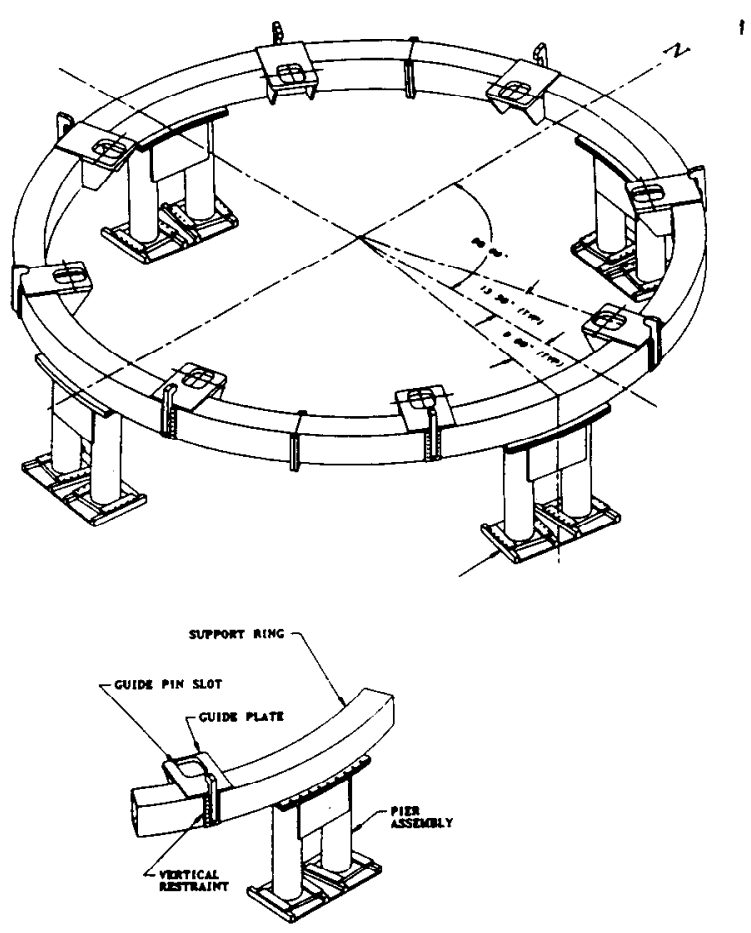

Fig. 12. Tokamak support structure.

tubing to valved connections in the test cell. The interspace is used for leak detection and can be pumped to mitigate the effect of a leak should one be detected.

During operation the space between the vacuum vessel inner and outer shells is filled with $150^{\circ} \mathrm{C}$ water at $80 \mathrm{psi}$. This water acts as a thermalizing medium for the fusion neutrons produced during deuterium operation and serves to maintain the vessel at $150^{\circ} \mathrm{C}$ for wall conditioning purposes. The water is circulated through the passive plates and inboard limiters and returns to the space between the double shells. Return piping exits the bottom of the double shell space and connects to the heating/cooling system. During a pulse a heat exchanger in the heating/cooling system removes the heat generated. For bakeout, the water is drained from the wall interspace and replaced with superheated steam at $350^{\circ} \mathrm{C}$. This steam bakes out the vessel walls and all vessel internal components. Wall coolant panels are provided inside the vacuum vesel to protect vacuum vessel surfaces with line-of-sight to the plasma from radiation heat loads from the plasma.

Radiation shielding is provided by water in the double walled vessel jacket and by lead oxide/boron carbide tiles on the outside of the vacuum vessel shell. The boron carbide absorbs thermal neutrons while the lead oxide provides gamma attenuation. In order to minimize the activation of peripheral components, the large horizontal ports (except the active NB ports) have integral shield plugs installed in them which provides shielding equivalent to the vacuum vessel shell with shield tiles. Around the vertical ports and vacuum pumping ducts, polyethylene is used to thermalize the neutrons. The polyethylene contains boron for neutron absorption and a high density material for gamma attenuation. The radiation shielding limits the nuclear heating of the cold $(4 \mathrm{~K})$ mass and facilitates hands-on maintenance outside the shield boundary.

The maintenance approach on TPX is to provide remote maintenance inside the vacuum vessel, but, facilitated by the shielding, rely mainly on hands-on maintenance outside the vacuum vessel. A preliminary dose map around the tokamak is provided in Fig. 14. A hot cell facility is provided for repair of activated components. All of the PF coils (except PF5,6/L) can be replaced without disassembly of the TF. In the event of a TF or lower PF coil failure, or if a major machine reconfiguration was in order, highly activated components inside the vacuum vessel would be removed remotely. After a 1 year cooldown period, the titanium vacuum vessel would cool down to where hands-on maintenance could be performed inside and the tokamak could be disassembled or reconfigured. This maintenance approach reduces the scope and cost of remote maintenance while providing substantial operating time in deuterium and valuable operational experience with remote maintenance.

The vacuum vessel is too distant from the plasma to provide passive stabilization for the vertical instability. High strength, copper alloy plates are mounted off the vacuum vessel inboard and outboard of the plasma, above and below the midplane as shown in Fig. 15. These plates slow the motion of the plasma down so that vertical position control can be effected with fast vertical position control coils located behind the outboard passive plates. The upper and lower passive plates are connected in a saddle configuration to facilitate development of the $20 \mathrm{~V}$ loop voltage for startup. The fast vertical position control coils are connected in a like manner.

\subsection{Plasma Heating and Current Drive Systems}

Plasma heating and current drive is provided by three systems: neutral beam (NB), lower hybrid (LH), and ion cyclotron (IC). In addition, the facility is capable 


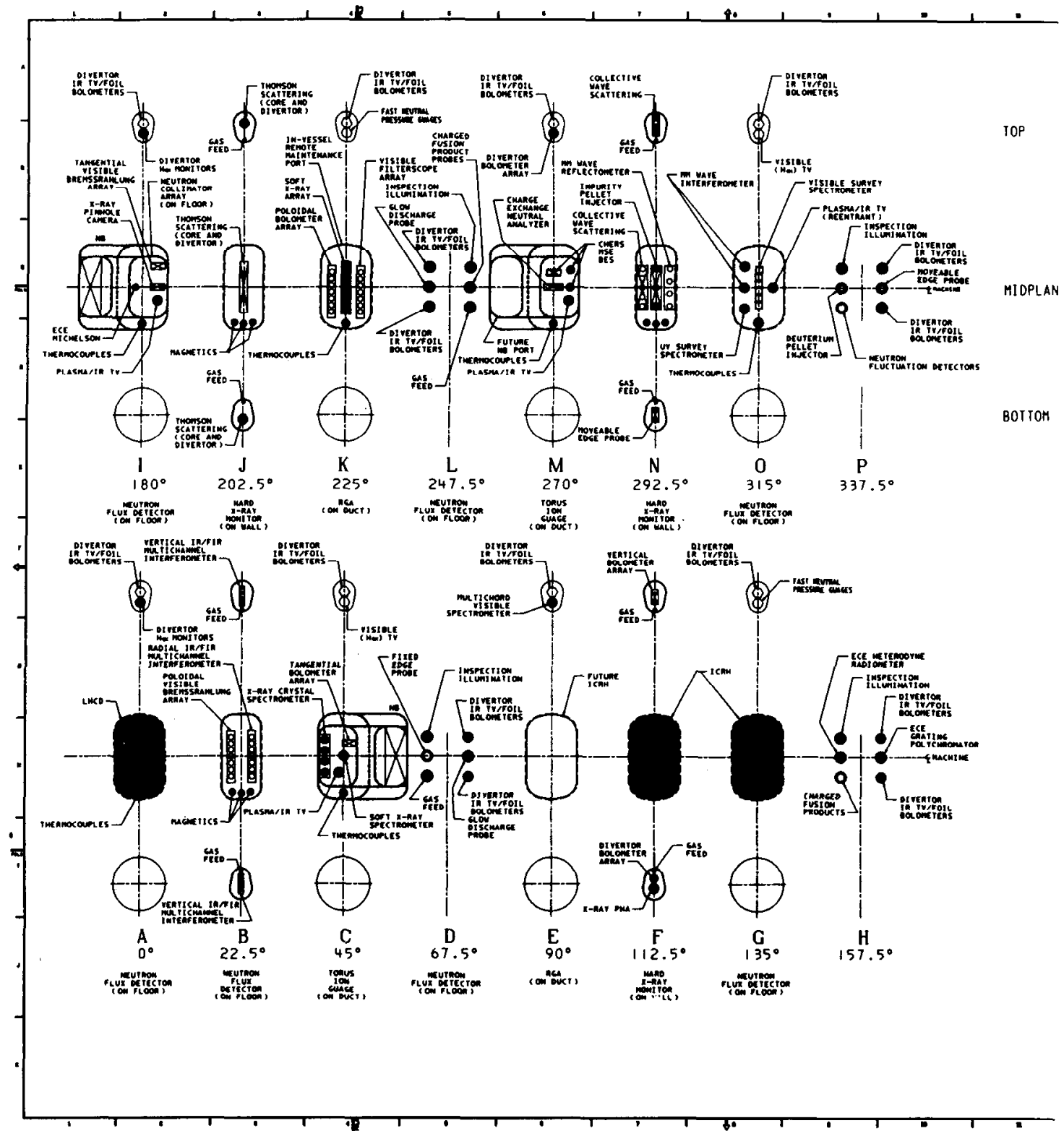

Fig. 13. TPX port allocations.

of accommodating 10MW of electron cyclotron (EC) heating.

The neutral beam injection system will consist initially of one modified TFTR beamline, its three longpulse ion sources, and the associated power supplies, and will be capable of injecting $8 \mathrm{MW}$ of neutral deuterium beam power into the plasma with an accelerating potential of $120 \mathrm{keV}$. The design reuses existing TFTR beam components wherever feasible, since this is a mature system which has functioned reliably for years.
The beamline is aimed so that the middle ion source is tangent to a major radius of $2.0 \mathrm{~m}$ for optimal current drive. The tokamak is configured to accommodate three beamlines (two co-directed beamlines and one counterdirected beamline) if necessary.

The ion sources, which were developed by Lawrence Berkeley Laboratory to be capable of 30 second pulses, will be reused with only minor modifications to improve the cooling of the electron dump. The liquid helium filled cryopanels, which form the basis of the 


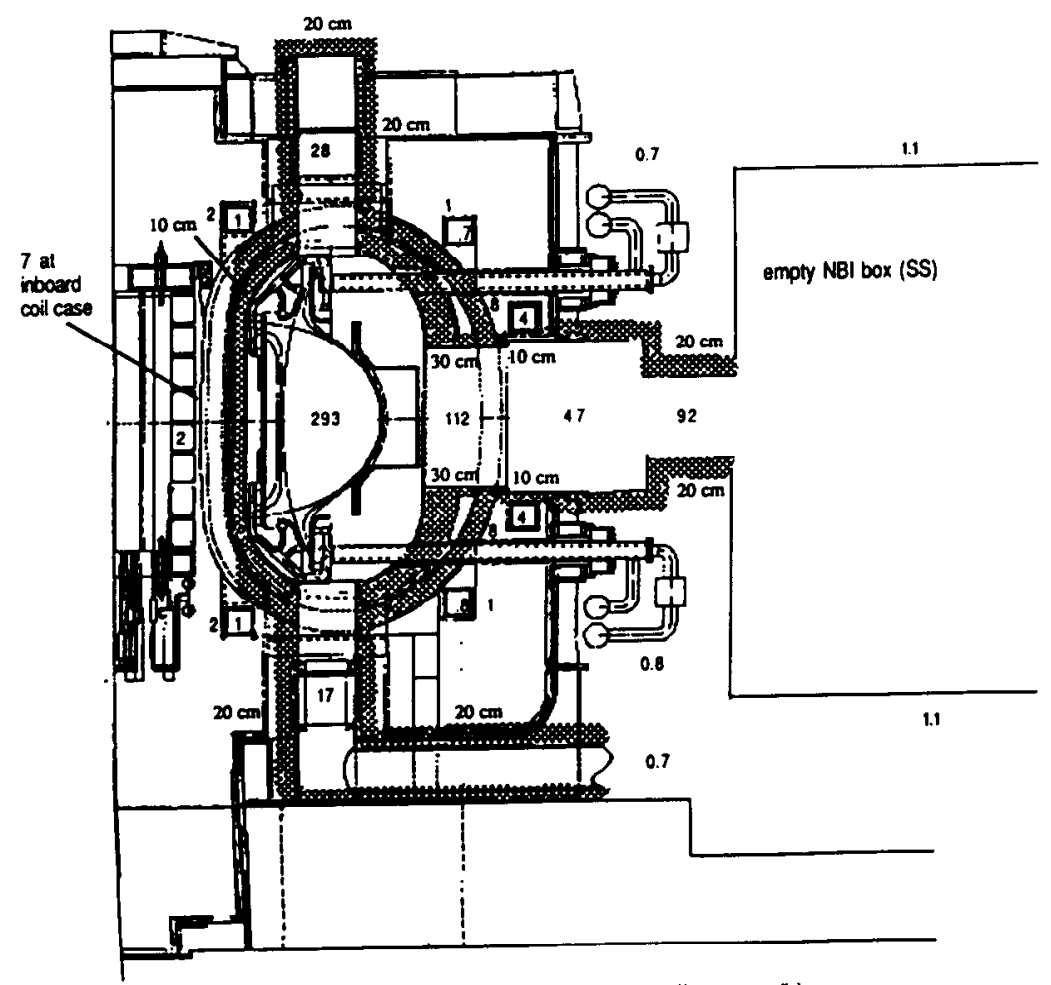

Fig. 14. TPX preliminary dose map (in mrem/h).

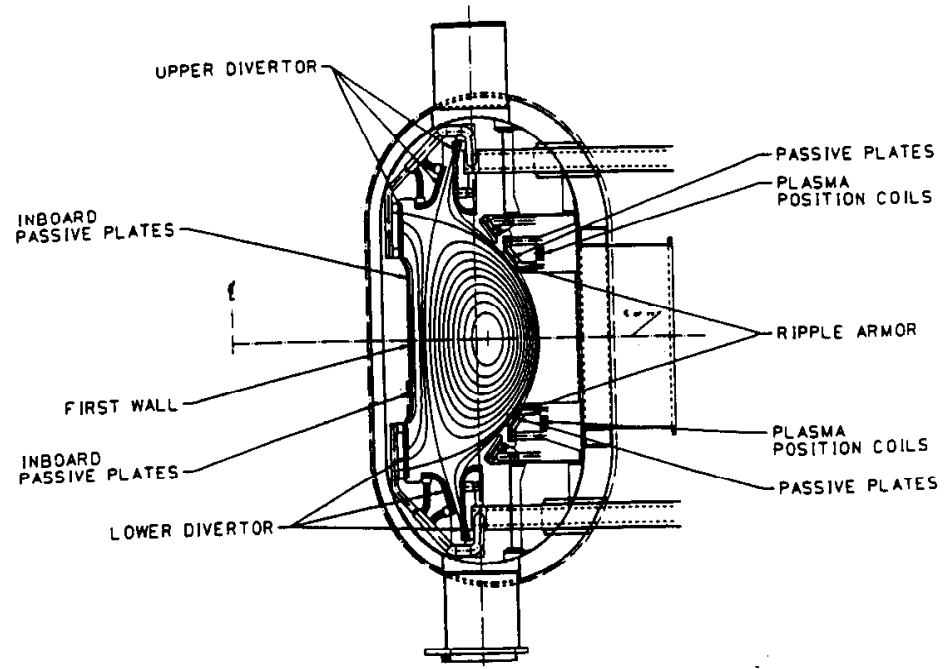

Fig. 15. VV internal components.

TFTR beam vacuum system, will be reused without modification. The present $1 \mathrm{~kW}$ liquid helium refrigerator will be retained, although the transfer lines will be upgraded. The three deflection magnets, which remove the residual ions from the beams after they emerge from the neutralizers, can be retained without modifications, 
as can their power supplies, which already run continuously. The large stainless steel beam box will also be retained, along with some of the mechanical components of the duct which connects the beam box to the tokamak vacuum vessel. An elevation view of the tokamak with a beam line attached is shown in Fig. 16.

Within the beamline, the extension of the pulse length to 1000 seconds will primarily have an impact upon the beam-impinged components. These will all be replaced with actively cooled hypovapotrons similar to ones developed for JET and its neutral beamlines. In particular, hypovapotrons will be used for the ion dumps, calorimctcrs, scrapers, neutralizers, and duct liners.

Large portions of the power supplies which drive the ion sources and accelerators will be reused with slight to moderate modifications, including the transformer/rectifiers, the tap changer transformers, the modulator/regulator tetrode tubes, the surge rooms, the switchyard breakers, and the decel supplies. Upgrades for steady state operation will be made to the gradient grid voltage dividers and to the arc and filament supplies.

The LH system provides $1.5 \mathrm{MW}$ of heating and current drive power and is capable of being upgraded to provide $3 \mathrm{MW}$ of LH power if necessary. The LH system is critical for providing current profile control which is effected by adjusting the launched wave number spectrum of the $\mathrm{LH}$ power.

The LH system consists of the following elements:

- Four (of the 12 existing) TFTR NB power supplies, modified to deliver the required voltages for the klystrons;

- Four $3.7 \mathrm{GHz}$ klystrons (with two output windows per klystron) and associated if drivers, which convert the DC power to if power;

- Eight lengths of WR284 waveguide, which carry the rf power from the klystron location to the TPX area;

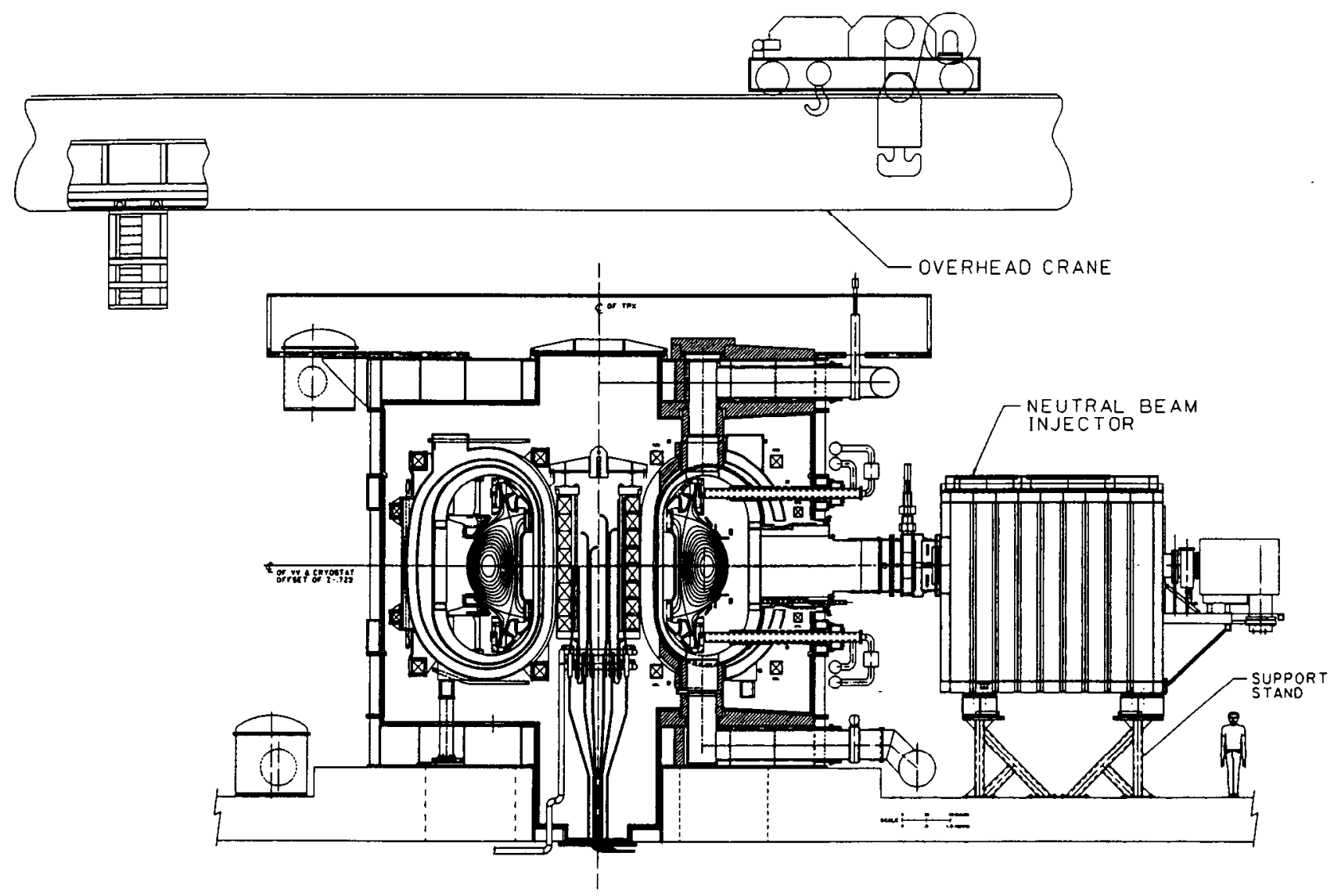

Fig. 16. TPX beamline elevation. 
- Two boxes with power splitting and phase control circuitry, which divide the eight power inputs into 32 independently phaseable outputs (more WR284 guides carry the if power to the launcher input);

- A radially moveable $\mathrm{LH}$ launcher, consisting of 128 waveguide elements, and a final splitter (mounted just outside the port) that divides the 32 inputs into 128 outputs;

- An instrumentation and control (I\&C) system to control and monitor the operation of the LH system.

The LH launcher (shown in Fig. 17) is installed in a large horizontal port and is radially moveable for plasma coupling. Radiation shielding is provided integral to the launcher to minimize activation of components on the tokamak periphery.

The IC system provides $8 \mathrm{MW}$ of heating and current drive power to the plasma through two adjacent large horizontal ports. The IC system can be upgraded to provide up to $18 \mathrm{MW}$ through three ports if necessary. The design must provide for long-pulse operation, the ability to tune and match the antennas to the varying plasma loads, and the ability to change the parallel wave number spectrum of the launched power over a range of frequencies and plasma loads.
The IC system consists of the following subsystems:

- HV supplies and power units which will be modified versions of present equipment now being used on TFTR;

- Four RF power units (also called transmitters), which convert the DC power to if power;

- RF transmission lines, which carry the rf power approximately $800 \mathrm{ft}$ from the present transmitter location to the TPX area;

- Tuning and matching circuitry, which adjusts the amplitude and phase of the currents in the antenna current straps as desired during plasma operation;

- Two antennas in two ports, each containing six current straps, to couple the if power to the plasma;

- An instrumentation and control (I\&C) system to control and monitor the operation of the IC system.

The IC launchers are installed in large horizontal ports at a fixed distance from the plasma edge. The launcher design is shown in Fig. 18 (sans Faraday shield). Coupling with the plasma is maintained by moving the plasma edge relative to the launcher. Radiation shielding is provided integral to the launcher to minimize activation of components on the tokamak periphery. The launcher fea-

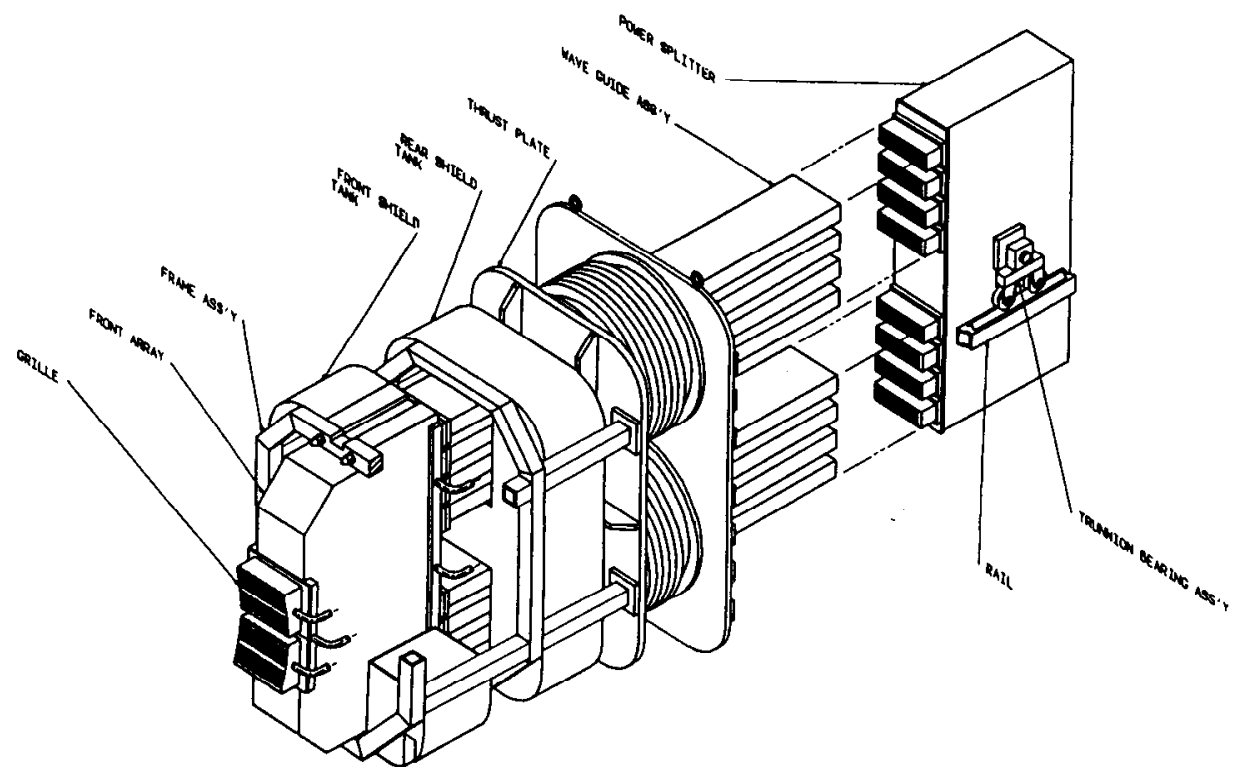

Fig. 17. LH launcher. 


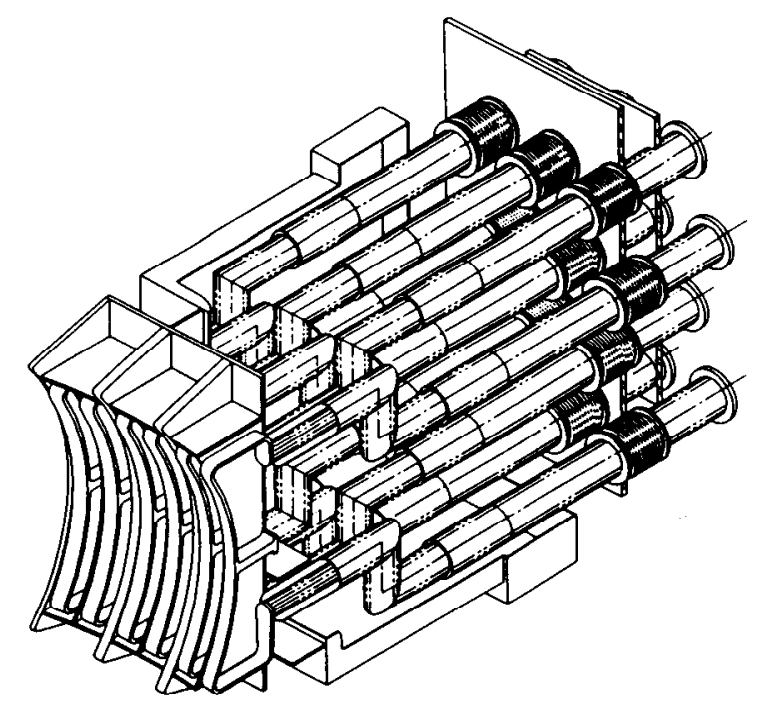

Fig. 18. IC launcher.

tures an Inconel Faraday shield. Inconel was selected as the Faraday shield material in spite of its poor activation characteristics because of its ease of fabrication and demonstrated reliability in existing tokamaks. To facilitate hands-on maintenance inside the vacuum vessel for the first two years of operation, a lead shield will be installed over the Faraday shield prior to entry of maintenance personnel.

\subsection{Power Handling and Particle Control Systems}

TPX is designed for operation with a double null divertor, although a single null capability has been retained. In addition, toroidal and poloidal limiters are provided for startup and to protect the passive stabilizers and equipment in the port regions from energetic particle fluxes during steady state operation and disruptions. High heat flux armor is provided for neutral beam shine-through and local heat loads due to ripple-trapped particles. The configuration of these plasma facing components is shown in Fig. 15.

Plasma facing components are designed to handle a maximum plasma heating power of $45 \mathrm{MW}$ under two sets of conditions: a high core radiation condition in which $50 \%$ of the plasma heating power is radiated in the plasma core and a low core radiation condition in which $80 \%$ of the plasma heating power is conducted into the scrape-off layer (SOL). The low core radiation condition drives the divertor design. The high core ra- diation condition drives the design of the other plasma facing components.

All plasma facing components use graphitic plasma facing materials and have steady state power removal capability. Depending on the level of heat flux, these components are either bolted or brazed Carbon-Carbon (C-C) composite tiles on water cooled structures. All plasma facing components are designed for complete remote maintainability and are made from low activation materials where possible. A brief summary of each plasma facing component follows:

- Divertor-The divertor provides for a single and double null configuration. It is divided into 16 toroidal sectors. Each sector is separable into two parts to facilitate remote handling: (1) inner plate with its water manifolds, (2) center and outer plate with its water manifolds. The target plates consist of high conductivity C-C composite tiles that are brazed to dispersion strengthened copper coolant tubes. A monoblock design combined with a coolant channel with a twisted tape insert and a $10 \mathrm{~m} / \mathrm{s}$ coolant velocity is used in the high heat flux regions. The target plates are designed for a peak heat flux of $15 \mathrm{MW} / \mathrm{m}^{2}$ but with radiative or gaseous divertor performance lower loads are expected.

- Inboard Limiter-The inboard limiter is composed of C-C composite tiles that cover both the copper inboard passive plates and the modular titanium alloy panels between these plates. Except in areas of neutral beam shine through, this component experiences modest heat loads and therefore consists primarily of bolted tiles. In the areas of neutral beam shine through a brazed tile design is employed.

- Outboard Toroidal Limiters-The outboard toroidal belt limiter consists of $\mathrm{C}-\mathrm{C}$ composite tiles that are bolted to the cooled copper inboard passive plates. This component only experiences the radation heat load from the plasma.

- Poloidal Limiters-At three discrete toroidal locations $0.5 \mathrm{~m}$ wide rail limiters are installed between the outboard toroidal limiters. These limiters are used primarily during startup, and experience only radiation heat load during steady state. They consist of C-C composite tiles that are bolted to cooled titanium support panels.

- Ripple Armor-This armor intercepts the trapped energetic particles that are drifting vertically in the ripple region. This armor consists of $\mathrm{C}-\mathrm{C}$ composite tiles brazed to copper heat sink armor 
sections located between the TF coils at the edges of the upper and lower outer passive plates.

- Neutral Beam Armor-This armor intercepts neutral beam depositions from shine through and faults. High heat flux areas are of the brazed tile to heat sink design. Lower heat flux areas use a bolted tile concept. Support plates are Titanium alloy.

Fueling is provided to the plasma with neutral beams (for core fueling) or with gas puffing (for edge fueling). The facility can accommodate a pellet injector if necessary. Particles leaving the plasma are exhausted through the divertor. A re-entrant divertor configuration with a baffle in the private flux region (shown in Fig. 15) is the baseline design. The divertor is designed to allow a gaseous or radiative target to be formed which would spread the heat flux out over a larger area, thus reducing the peak local heat flux. Gas can be puffed directly into the divertor region to facilitate formation of a gaseous or radiative target.

Neutral particles in the divertor region are exhausted through the vertical ports which are connected to the vacuum pumping ducts. Auxiliary baffles are provided to minimize the flow of neutral particles back to the plasma. Sixteen cryopumps are provided for vacuum pumping. A variable aperture is provided in front of the cryopumps to control the pumping speed and the pressure in the cryopumps. Each cryopump can store 25,000 torr-l of hydrogen or deuterium before needing to be regenerated. A pump can be regenerated in 2.5 hours. For normal operation with throughputs less than 100 torr-l/s, eight pumps are used while the other eight are being regenerated. For higher throughputs, all sixteen pumps would be used with a corresponding reduction in duty factor.

TPX is designed to provide glow discharge cleaning (GDC) under bakeout conditions (at $350^{\circ} \mathrm{C}$ ) and between pulses (at $150^{\circ} \mathrm{C}$ ). For $\mathrm{GDC}$, vacuum pumping is provided by turbopumps which are connected to the vacuum pumping ducts.

The effluent from the torus is processed through the TFTR Tritium Purification System to remove tritium generated by DD fusion reactions prior to release up the stack in accordance with ALARA principles. Effluents from other vacuum systems such as the neutral beam and cryostat vacuum systems are released directly up the stack at " $D$ " site.

\subsection{Maintenance Systems}

In general, maintenance to systems located external to the radiation shielding will be accomplished with hands on operations. Maintenance to systems located internal to the radiation shielding will be accomplished remotely once material activation exceeds exposure limits imposed by PPPL administrative limits. The TFTR hot cell facility will be used for repair of activated components.

One horizontal port is provided for primary access of the maintenance equipment into the vacuum vessel. Shielding is integrated into the maintenance systems at the port area to permit personnel access in the test cell during maintenance operations and during transfer of contaminated/activated components to the hot cell.

Some components attached to the horizontal ports and subject to neutron streaming may become mildly activated and require special precautions during maintenance to limit personnel exposure. Examples are diagnostic equipment and the NB torus isolation valve. Maintenance of these components will be handled on a case-by-case basis. The typical approach to provide for maintenance of these items will be specialized designs that include localized shielding, the use of long handled tools, and shielded access to fasteners and hold-downs.

The basic components of the in-vessel remote manipulator system are a transfer manipulator located at Port $O$ and two in-vessel vehicles. The transfer manipulator moves one of the in-vessel vehicles through the port into the center portion of the vessel, rotates the vehicle into an upright oricntation, and positions it on the titanium rails in the vessel. The second vehicle is deployed onto the rails in a similar manner. One vehicle is equipped with a power arm manipulator and the other equipped with a dexterous servo manipulator arm. An elevation view of the tokamak with the transfer manipulator and in-vessel vehicle shown is provided in Fig. 19.

Transfer to and from the vessel of tooling, removed components, and replacement components is accomplished by the transfer manipulator. Activated or contaminated components removed from the vessel are placed in a shielded transfer container for transport to the hot cell.

The power arm manipulator does not have the capacity to lift a divertor module. To replace a divertor module, the transfer manipulator deploys the elevator platform and the divertor module handling device into the vessel and positions in onto the elevator carriages of the two in-vessel vehicles, locking the two vehicles together. The elevator carriages provide the vertical lift capacity to handle a divertor module.

The divertor module handling device provides the articulation to manipulate a module from its mounting location and clear the passive stabilization plates, and the in-vessel vehicles provide the motive power to trans- 


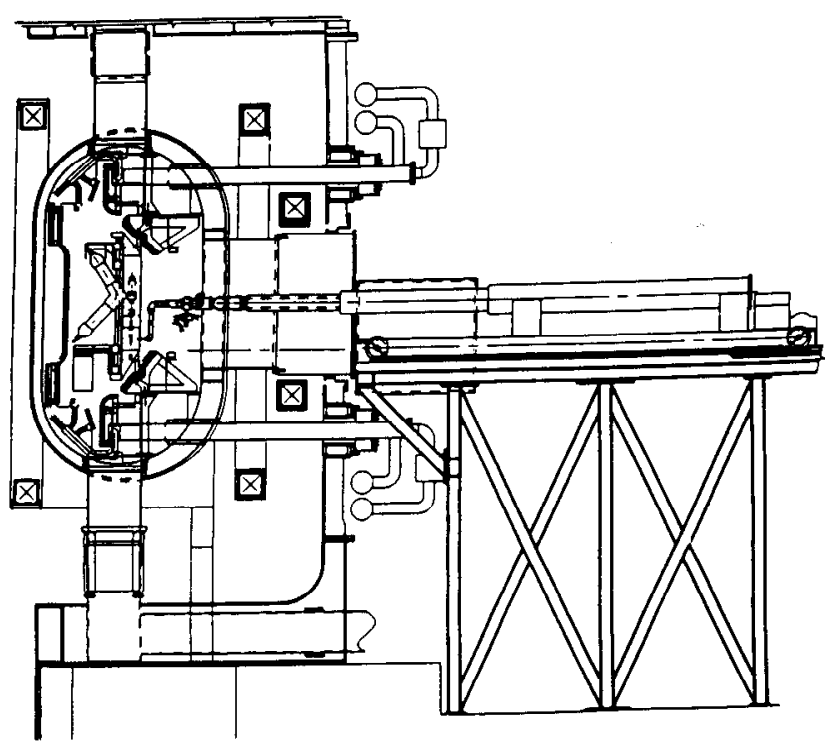

Fig. 19. In-vessel manipulator.

port the module toroidally to Port $\mathrm{O}$. At this location, the transfer manipulator reaches into the vessel, grasps the module, retracts it from the vessel, and places it in the shielded transfer container. Replacement modules are reinstalled in a reverser procedure.

Special remote tools required for maintenance of system components will be provided by each system. The remote maintainability of equipment likely to require remote maintenance during the life of the machine will be demonstrated on mockups prior to final design.

\subsection{Data Systems}

An instrumentation and control (I\&C) system is provided for coordination of operations and the acquisition, archiving, and display of experimental data. The I\&C system will be designed for steady state operation. The operations environment will provide real-time display of information and allow changes to be implemented during the course of a discharge.

The national perspective of TPX requires that researchers located at the TPX site and at other laboratories and universities have access to the facility in support of the remote operation, physics planning, analysis, and coordination of experimental objectives. A distributed computing environment spanning several geographically separate sites, is envisioned for TPX. The sites will be connected via a high performance, wide area computer network, with sufficient bandwidth to allow transmission of information including file transfers, graphical displays, and video information.

\subsection{Power System}

The electrical load for TPX has the following characteristics:

- The power demand of the TF system is quasicontinuous and very small, amounting only to the lead losses during steady state operation.

- The power demand of the PF system is fairly large during plasma ramp-up and ramp-down, but is minimal once the plasma has been established, amounting only to the lead losses plus small additional loading due to position and shape adjustment and variation in plasma confinement requirements.

- The power demands for fast vertical position control and field error correction are modest.

- The major power demand is for the auxiliary heating systems which operate for long periods once the plasma has been established.

The power systems design has the following significant features:

- The TF system is powered by a new low voltage, high current converter powered directly from the utility grid power source.

- The PF system is powered using existing thyristor converters. These are supplied from one of the two existing motor-generator sets. The excitation of the generator is controlled such that the full converter output voltage range is available while ramping the plasma current. Reduced converter output voltage range is available once the plasma has been established. This scheme reduces the reactive power loading of the converters, reduces the harmonic content of the converter output voltage, and increases the precision of control resolution when low voltage is demanded.

- The fast vertical position control and field error correction coils are powered using new power supplies which are powered directly from the utility grid power source.

- The auxiliary heating systems are powered directly from the utility grid power source, through a new $138 \mathrm{kV}$ substation located at D-site. This substation is adjacent to the load, to minimize transmission costs and power loss. The power levels arc within the capability of the grid con- 
nection. As more auxiliary heating power is added to the machine modest modifications to the grid connection will be required. The energy demanded during a pulse far exceed that which would be available from motor generator sets, so that the use of utility power is essential.

Quench protection of superconducting coils is a critical feature in the power systems. If a quench of the superconductor is detected, then the energy stored in the magnetic field of the magnet(s) must be rapidly dumped in the external circuit in order to avoid overheating the quenched region of the magnet. However, the dump voltage must not exceed the voltage rating of the individual coils. The energy dump feature must be extremely reliable to avoid potential coil damage.

In order to provide the required energy dump protection, redundant $D C$ circuit breakers with shunt dump resistors are utilized in every magnet circuit loop. Operation of either one of the two devices in each loop will result in a current decay, even from the highest permitted current level, such that the joule heating in the quenched magnet is within limits.

\subsection{Cryogenic and Water Cooling Systems}

TPX facilities include liquid helium and liquid nitrogen systems, water cooling systems, buildings, and site improvements. The cryogenic helium system for TPX is required to cool and maintain the superconducting magnets near $4 \mathrm{~K}$ during operations. Additionally, the system will provide refrigeration for the cryogenic vacuum pumps. Heat loads include neutron heating, eddy current heating, thermal radiation from warm surfaces, conduction along supports, and helium flow to support gas-cooled magnet leads.

Helium refrigeration will be provided by an existing $11 \mathrm{~kW}$ refrigerator. The refrigerator was previously used on the mothballed Mirror Fusion Test Facility (MFTF) at Livermore, California. It includes 5700 horsepower of compressors, a cold box containing heat exchangers and expanders, and associated controls. It will be modified to deliver forced-flow supercritical helium to the magnets at about $0.90 \mathrm{MPa}$.

A 60,000 liter liquid helium dewar, also surplus equipment from MFTF, will be modified to serve as a liquid-helium storage system and buffer volume for adsorbing pulsed loads from neutron and eddy current heating. A novel system of heat exchangers and valving at the dewar will ensure the refrigerator receives the steady load required for efficient operation.

A liquid-nitrogen system supported by truck-deliv- ered liquid nitrogen is also a part of the cryogenic system. Its purpose is to provide liquid nitrogen for thermal shields through out the facility.

The cryogenic system major components will be housed in a new $1000 \mathrm{~m}^{2}$ building about $25 \mathrm{~m}$ west of the TPX building, shown in Fig. 20. An additional 1000 $\mathrm{m}^{2}$ of outdoor space is used for gaseous-helium tanks, liquid-nitrogen storage tanks, and truck-trailer access.

The TPX water cooling system utilizes existing cooling towers and auxiliaries at PPPL. These will be augmented by water storage tanks to provide for long pulse (1000 second) operation. The cooling system provides cooling water for the heating systems, the TF and $\mathrm{PF}$ rectifiers and bus, the cryogenic refrigerators, the internal vacuum vessel components, and the MG set. $350^{\circ} \mathrm{C}$ steam will be provided to the vacuum vessel for bakeout. $150^{\circ} \mathrm{C}$ water will be required for the vacuum vessel and certain internal components. High heat flux components internal to the vacuum vessel will be provided $35^{\circ} \mathrm{C}$ cooling water during a pulse, and $150^{\circ} \mathrm{C}$ water between pulses. $35^{\circ} \mathrm{C}$ tower conling water will be required for the cryogenic refrigeration system and the MG set.

\subsection{Assembly, Installation, and Test}

Assembly and test begin with the preparation of the assembly areas, the mockup building and the test cell, and end with the achievement of a first plasma 2 years later. The major machine components are assembled during the first year. The second year can be roughly divided into three equal periods. During an initial test period the cryogenic systems are tested, and low level power is delivered to the TF coils for magnetic field mapping. This is followed by the installation of the internal vacuum vessel components. In parallel with these activities the diagnostics are installed, the neutral beamline reassembled, and RF launchers and antennae completed. The final period consists of the integrated systems tests culminating in first plasma.

The first components to be delivered to the assembly area are the segments which make up the cryostat base, cylindrical sections, and dome. The cryostat base is assembled on the machine foundation together with the machine support structure. The remainder of the cryostat is assembled, vacuum tested, and then dismantled to prepare for the TF quadrant assembly. In parallel, shielding tiles and superinsulation are installed on the vacuum vessel segments in the mockup building. TF two-coil modules are delivered and assembled into quad rants in the same location. The assembly of the shielded 


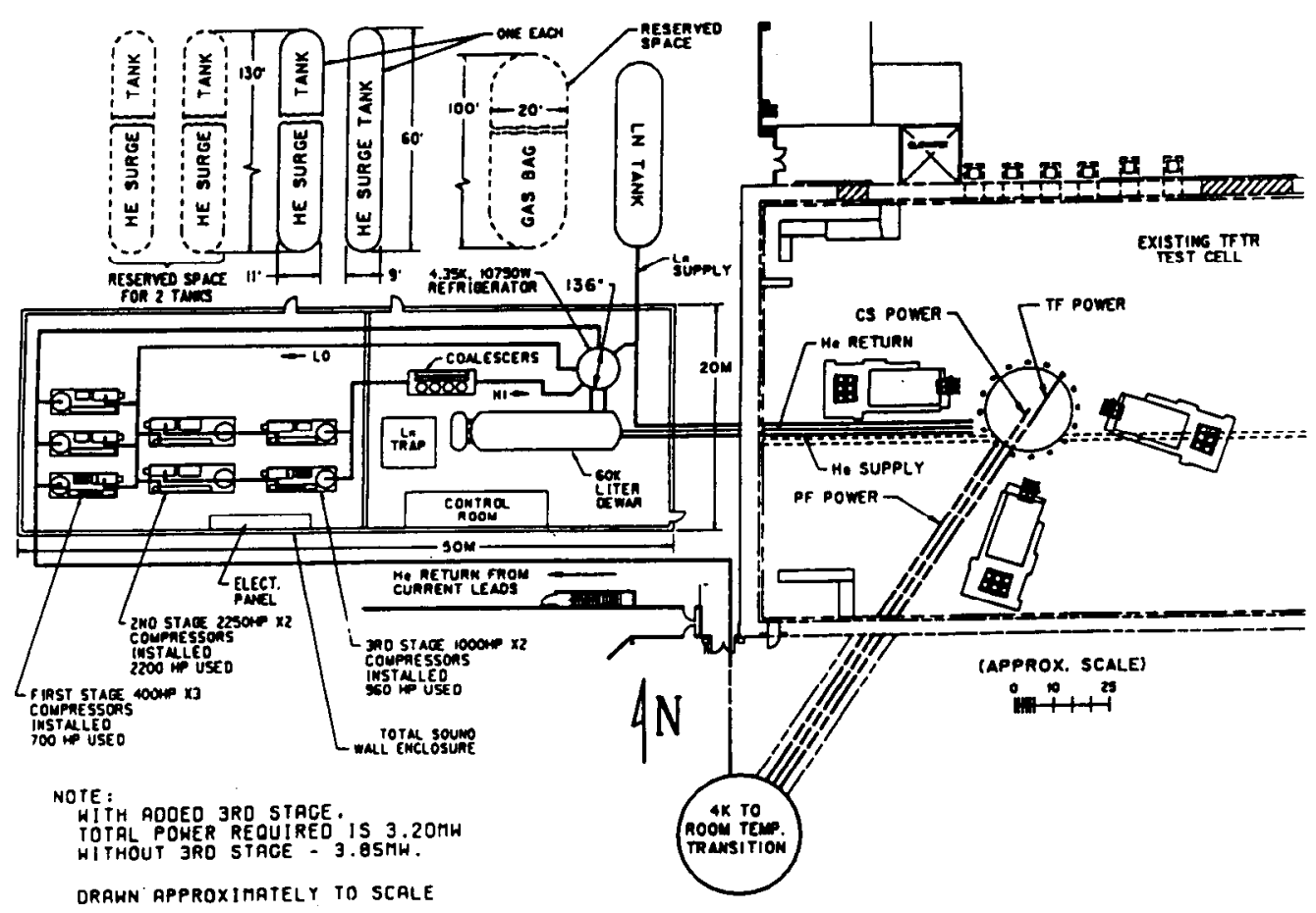

Fig. 20. Cryogenic system major components.

TPX Organization

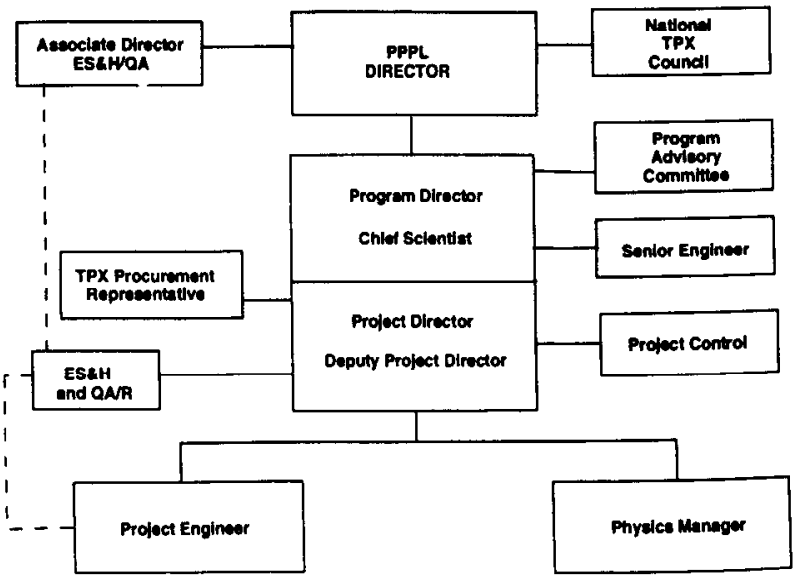

Fig. 21. TPX organization chart.

vacuum vessel segment and a $\mathrm{TF}$ quadrant also takes place in the mockup building. As each quadrant is completed, it is delivered to the test cell and erected onto the support structure. When all of the TF quadrants have been assembled, they are aligned and welded together.
At the completion of the TF quadrant welding, the vacuum vessel segments are positioned and welded. The PF coils are then installed followed by the assembly of the PF solenoid. The assembly and welding of the vacuum vessel ports must be integrated with the re-assembly of the cryostat. Meanwhile, the installation of TF and PF jumpers, leads, bus, and coolant lines will take place.

Following leak check and repair welding of the vacuum vessel, the initial testing phase begins. This includes a vacuum check of the cryostat, followed by cryogenic cooldown. A low level power test, developing a field of up to $1 \mathrm{~T}$, leads to magnetic field mapping. Fiduciary points established during magnetic field mapping form the basis for the mechanical location of the intcrnal components. The installation of these components (divertors, limiters, passive plates, armor, plasma position coils, etc.) is on the critical path of the assembly operations leading to the final phase of the systems integration tests. The culmination of these tests will be the achievement of a first plasma.

\section{ORGANIZATION}

The TPX Project draws upon major DOE laboratories, universities, and many industrial subcontractors 
Table VI. TPX Conceptual Cost Estimate Summary

\begin{tabular}{|c|c|c|}
\hline WBS & Description & $\operatorname{Cost}^{a}$ \\
\hline 1 & Tokamak systems & Total $=\$ 224.4$ \\
\hline 11 & Plasma facing components & $\$ 48.7$ \\
\hline 12 & Vacuum vessel & $\$ 21.7$ \\
\hline 13 & TF magnet system & $\$ 70.6$ \\
\hline 14 & PF magnet system & $\$ 45.9$ \\
\hline 15 & Cryostat & $\$ 9.2$ \\
\hline 16 & Tokamak suppọtt structure & $\$ 1.3$ \\
\hline 17 & Tokamak assembly and testing & $\$ 14.0$ \\
\hline 18 & Tokamak radiation shielding & $\$ 9.8$ \\
\hline 19 & Field error/fast vertical position control coils & $\$ 3.2$ \\
\hline 2 & Auxiliary heating and current drive & Total $=\$ \$ 50.7$ \\
\hline 21 & Neutral beams & $\$ 18.5$ \\
\hline 22 & $\mathrm{ECH} / \mathrm{ECCD}$ & $\$ 0.0$ \\
\hline 23 & ICH/FWCD & $\$ 19.1$ \\
\hline 24 & LHH/LHCD & $\$ 13.1$ \\
\hline 3 & Fueling and vacuum systems & Total $=\$ 11.8$ \\
\hline 31 & Fueling storage and delivery & $\$ 0.8$ \\
\hline 32 & Pellet injector & $\$ 0.0$ \\
\hline 33 & Radiation monitoring and tritium cleanup & $\$ 0.3$ \\
\hline 34 & Vacuum pumping systems & $\$ 10.7$ \\
\hline 35 & Tritium systems & $\$ 0.0$ \\
\hline 4 & Power systems & Total $=\$ 25.5$ \\
\hline 41 & AC power systems & $\$ 5.4$ \\
\hline 42 & TF power systems & $\$ 3.0$ \\
\hline 43 & PF power systems & $\$ 12.4$ \\
\hline 44 & Fast vertical position control coils power systems & $\$ 1.3$ \\
\hline 45 & Field error correction coils power systems & $\$ 0.6$ \\
\hline 46 & Control systems & $\$ 2.6$ \\
\hline 47 & Dummy load & $\$ 0.2$ \\
\hline 5 & Remote maintenance systems & Total $=\$ 20.6$ \\
\hline 51 & In-vessel systems & $\$ 9.5$ \\
\hline 52 & Ex-vessel systems & $\$ 4.1$ \\
\hline 53 & Tooling and miscellaneous & $\$ 5.2$ \\
\hline 54 & Hot cell equipment & $\$ 1.8$ \\
\hline 6 & Plasma diagnostics and data systems & Total $=\$ \mathbf{3 8 . 2}$ \\
\hline 61 & Central I and $\mathrm{C}$ & $\$ 11.3$ \\
\hline 62 & Plasma diagnostics & $\$ 26.9$ \\
\hline 7 & Facilities & Total $=\$ \mathbf{2 2 . 6}$ \\
\hline 71 & Building modifications or new buildings & $\$ 3.3$ \\
\hline 72 & Cryogenic equipment & $\$ 12.3$ \\
\hline 73 & Water cooling & $\$ 7.5$ \\
\hline 74 & Radiation shielding & $\$ 0.2$ \\
\hline 8 & Preparations for operations & Total $=\$ 6.7$ \\
\hline 9 & Project support & Total $=\$ 55.6$ \\
\hline 91 & Project management and administration & $\$ 9.7$ \\
\hline 92 & Safety documentation & $\$ 2.1$ \\
\hline 93 & Project physics & $\$ 10.1$ \\
\hline 94 & Systems integration & $\$ 19.8$ \\
\hline \multirow[t]{4}{*}{95} & Construction management & $\$ 13.9$ \\
\hline & Subtotal & $\$ 456.1$ \\
\hline & Contingency & $\$ 82.8$ \\
\hline & Total project costs (TPC) & $\$ 538.9$ \\
\hline
\end{tabular}

- Costs are in FY93 MS and represent the current baseline OPEX and PACE costs. Contingency costs have been combined and added explicitly to the subtotal of system costs without contingency. 


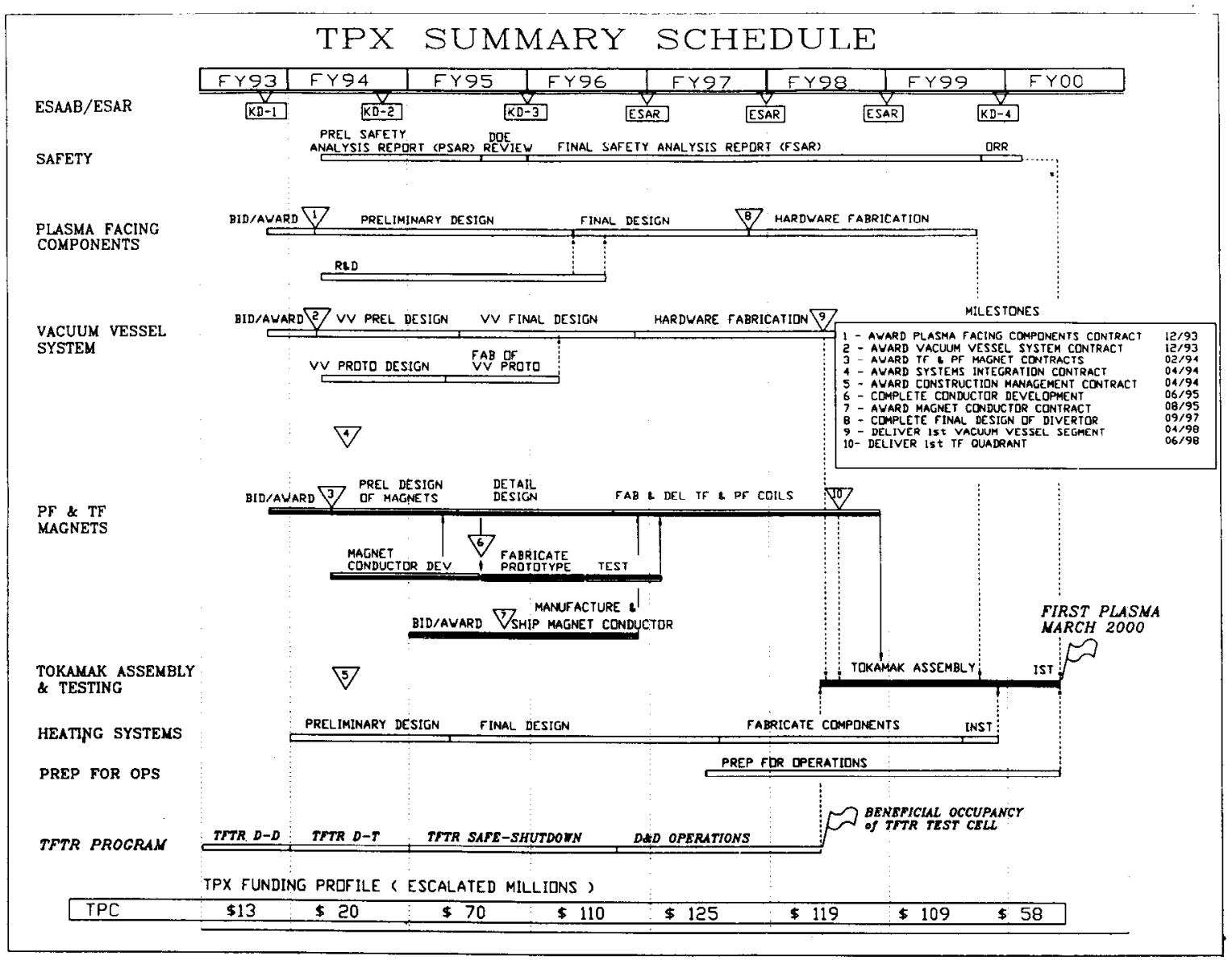

Fig. 22. TPX summary schedule.

for Physics, R\&D, and design support. PPPL retains overall responsibility for the project.

The TPX Conceptual Design has been developed and supported by a nation-wide team of physicists and engineers that includes the following major participants:

- Princeton Plasma Physics Laboratory (PPPL)

- Lawrence Livermore National Laboratory (LLNL)

- Oak Ridge National Laboratory (ORNL)

- Massachusetts Institute of Technology (MIT), Plasma Fusion Center

- Idaho National Engineering Laboratory (INEL)

- Los Alamos National Laboratory (LANL)

- Argonne National Laboratory (ANL)

- Ebasco Industrial Team consisting of: -Ebasco Services, Inc.

-McDonnell Douglas, Missile Systems Company

-Rockwell International
- GA Technologies, Team consisting at:

-General Atomics, Inc.

-McDonnell Douglas, Missile Systems Company

- Grumman Aerospace Corporation

- Universities:

-UCLA

-University of Wisconsin

-Columbia University

-New York University

The line of responsibility for TPX runs from the Department of Energy's (DOE) Office of Fusion Energy (OFE) to the DOE Chicago Field Office (CH) and the DOE Princeton Area Office (PAO) to the Directory of PPPL. The TPX Project Organization is under the PPPL Director as shown in Fig. 21.

For the TPX Project, the authority of the Director of PPPL flows to the two key positions; the TPX Pro- 
gram Director and the TPX Project Director. The TPX Program Director has management responsibility for developing the mission, physics objectives and program requirements of TPX, and reports to the PPPL Director. The TPX Project Director has management responsibility for project execution, including engineering design, physics/engineering interface, and resource management. On project issues significantly affecting the TPX program, the Project Director reports to the Program Director, and the senior TPX management will work as a team to resolve any related issues. On issues related to the execution of the TPX Project, the Project Director reports to the PPPL Director. The TPX Program Director and TPX Project Director are assisted by a senior TPX Project Management team that consists of the following positions:

- Chief Scientist

- Senior Engineer

- Deputy Project Director

- Project Physics Manager

- Project Engineer

- Project Control Manager

The TPX Chief Scientist is a senior advisor on scientific issues, and participates in technical and management decisions related to the mission, objectives, physics requirements, and design. He assists the Program Director to provide a liaison to the fusion physics community, to convey the mission and objectives of the device to the community, and to coordinate physics R\&D within the community in support of TPX. The Senior Engineer advises both the TPX Program Director and TPX Project Director on programmatic and project-related engineering matters.

The Deputy Project Director is the next senior line manager in the TPX Project Organization. He assists the Project Director in performing his functions and acts as the Project Director whenever the Project Director is not available. Additionally, he supports the TPX Project Director in his project advocacy role.

The Project Physics Manager and the Project Engineer head the main elements of the TPX line organization. Project Physics is led by the Project Physics Manager who is responsible for the development of the physics design requirements and analysis activities in support of TPX design and construction. The Project Engineer is responsible for developing the engineering design of TPX, optimized to meet the TPX Mission and design requirements, within the TPX Project cost and schedule objectives.

The Project Control Manager and his organization are responsible for all project support operations. He reports directly to the TPX Project Director in a staff function, but provides direct support to all levels of the TPX organization.

\section{COST AND SCHEDULE}

\subsection{Cost}

The TPX Conceptual Design cost estimate establishes the baseline $R \& D$, operating, and construction costs associated with the Project. It consists of the Project and Capital Equipment (PACE) direct and indirect costs estimated for the engineering, procurement, and installation of new equipment, the construction of new facilities, and any modifications to existing equipment facilities. The sum of all the PACE costs form the Total Estimated Cost (TEC) for the Project. The sum of the TEC and operating (OPEX) costs such as Conceptual Design, $R \& D$, and mockups/prototypes to be incurred prior to TPX being placed in operation, form the Total Project Cost (TPC). The DOE funding authorization usually consists of both PACE and Operating (OPEX) funding.

Engineering, hardware, installation, and modification costs have been estimated for each subsystem at the WBS element level 4. These direct estimates are substantiated by catalog pages, vendor letters and memos of telephone quotes, etc. In addition, each subsystem manager has prepared a cost profile sheet for their subsystem. These profiles were then used to project escalated costs using the DOE provided escalation rates for DOE-ER projects.

Project support costs such as project management, safety documentation, physics, systems integration, and construction management were estimated based on the level of resources required to support hardware-related work. Applicable burdens and overheads were applied in accordance with written agreements with DOE, including incremental indirect rates for construction-related costs. Project support costs were then added to the other project costs to obtain the TPX estimate.

The subsystem managers evaluated the uncertainties in the PACE estimates and developed a contingency and supporting rationale. These are summarized as supporting "Contingency," and added to the "Subtotal" to produce the TPC shown in Table VI, TPX Conceptual Cost Estimate Summary. 


\subsection{Schedule}

Individual schedules for each subsystem element were developed by the subsystem managers and integrated in the PPPL PMS Network Scheduling system. Supporting R\&D activites were included. The TPX Summary Schedule is provided in Fig. 22.

\section{REFERENCES}

1. E. A. Lazarus, and the DIII-D Team. Dependence of $\beta \cdot \tau$ on plasma shape in DIII-D. Presented at 20th EPS Conference on Controlled Fusion and Plasma Physics, July 26-30, 1993, Lisboa, Portugal. To be published in Proceedings.

2. F. Najmabadi, et al. (1991). IAEA Würzburg, Germany, 1992/
Paper G-1-1-1(R); J. Wesley et al. (1991). IAEA Würzburg, Germany, 1992/Paper F-1-2; Y. Seki et al. (1991). Rep. JAERI-M 91081 , JAERI, Naka.

3. N. Inoue, et al. (1992). IAEA Würzburg, Germany, Paper G-1-4.

4. S. Texter, et al. (1993). Phys. Lett. A 175, 428; R. Kaita, et al. (1992). IAEA Würzburg, Germany, PBX-M post-deadline paper.

5. M. Kikuchi, (1990). Nucl. Fusion 30, 265; R. W. Conn, F. Najmabadi et al. (1990). ARIES-I, A Steady-State First-Stability Tokamak Reactor with Enhanced Safety and Environmental Features. 13th Int. Conf. on Plasma Physics and Controlled Nuclear Fusion Research, Washington, D.C., October 1-6, Paper IAEA-CN-53/H1.4.

6. N. A. Uckan, et al. (1990). ITER Physics Design Guidelines: 1989, ITER Documentation Series No. 10, IAEA, Vienna.

7. R. J. LaHaye, A. W. Hyatt, and J. T. Scoville (1992). Non-Linear Instability to Low $m, n=1$ Error Fields in DIII-D as a Function of Plasma Fluid Rotation and Beta. General Atomics Report GAA20824, May, to be published in Nucl. Fusion.

8. T. Petrie, et al. (1992). Divertor heat flux reduction by $\mathrm{D}_{2}$ injection in DIII-D. Bull. Am. Phys. Soc. 37, 1565 (abstract). 